\title{
Halting eternal acceleration with an effective negative cosmological constant
}

\author{
V.F. Cardone \\ Dipartimento di Fisica "E.R. Caianiello", Universitá di Salerno, via S. Allende, \\ 84081 - Baronissi (Salerno), Italy \\ R.P. Cardenas, Y. Leyva Nodal \\ Departamento de Fisica, Universidad Central de Las Villas, Santa Clara, CP, \\ 54830 - Villa Clara, Cuba
}

\begin{abstract}
In order to solve the problem of eternal acceleration, a model has been recently proposed including both a negative cosmological constant $\Lambda$ and a scalar field evolving under the action of an exponential potential. We further explore this model by contrasting it against the Hubble diagram of Type Ia supernovae, the gas mass fraction in galaxy clusters and the acoustic peak and shift parameters. It turns out that the model is able to fit quite well this large dataset so that we conclude that a negative $\Lambda$ is indeed allowed and could represent a viable mechanism to halt eternal acceleration. In order to avoid problems with theoretical motivations for both a negative $\Lambda$ term and the scalar field, we reconstruct the gravity Lagrangian $f(R)$ of a fourth order theory of gravity predicting the same dynamics (scale factor and Hubble parameter) as the starting model. We thus end up with a $f(R)$ theory able to both fit the data and solve the problem of eternal acceleration without the need of unusual negative $\Lambda$ and ad hoc scalar fields.
\end{abstract}

PACS numbers: 04.50.+h, 98.80.-k, 98.80.Es

\section{Introduction}

Recent astrophysical data, from the Hubble diagram of Type Ia Supernovae (SNeIa) [1, 2] to the measurement of the anisotropy and polarization spectra of the cosmic microwave background radiation (CMBR) [3, 4] and large scale structure data [5], point out towards a picture of the universe unexpected only few years ago. According to this new scenario, the universe is spatially flat, undergoing a phase of accelerated expansion and dominated by a smoothly distributed negative pressure fluid dubbed dark energy. Although a lot of candidates has been proposed for this mysterious component [6], the concordance $\Lambda \mathrm{CDM}$ model [7] made out of cold dark matter (CDM) and the Einstein cosmological constant $\Lambda$ stands as the best fit to a wide range of different astrophysical data $[8,9,10]$. Notwithstanding its observational merits, the $\Lambda \mathrm{CDM}$ scenario is seriously

$\ddagger$ Corresponding author: winnyenodrac@gmail.com. 
plagued by the well known coincidence and fine tuning problems which are the main motivations to look for alternative models.

Although as a classical (general relativistic) field theory the concordance model is fairly simple, a universe presently dominated by a positive $\Lambda$ term is on the contrary quite difficult to understand from a quantum theory viewpoint. Indeed, since matter and radiation energy density fades away as $a^{-3}$ and $a^{-4}$ respectively (with $a$ the scale factor), the universe turns out to be asymptotically dominated by the vacuum energy accounted for by the positive $\Lambda$. As a consequence, the universe asymptotically enters a de Sitter phase with the scale factor growing exponentially and a never ending accelerated expansion. Let us then consider the definition of cosmic horizon as:

$$
\delta \propto \int_{t_{0}}^{t_{\text {end }}} \frac{c d t}{a(t)}<\infty
$$

with $t_{0}$ and $t_{\text {end }}$ the present day age of the universe and its age at the end of time (i.e., $a\left(t_{\text {end }}=0\right)$. Note that, while for a closed universe $t_{\text {end }}$ takes a finite value, this is not for open and spatially flat models $\left(t_{\text {end }} \rightarrow \infty\right)$. For a de Sitter like universe, $\delta$ takes a finite value so that a cosmic horizon appears. It is worth stressing that this problem is not strictly related to $\Lambda$, but turns out to be a consequence of eternal acceleration. Should we replace $\Lambda$ with a quintessence scalar field, the universe should still be eternally accelerated finally reaching a de Sitter phase and hence again a finite cosmic horizon.

The presence of a cosmic horizon implies that it is not possible to define pure state vectors of quantum asymptotic states. As a consequence, in a de Sitter background spacetime, it is not possible to define a gauge invariant scattering matrix $\mathcal{S}$. First studied in [11], this problem is known as superexpansionary divergence in the context of quantum field theory (QFT) and is particularly troublesome for the formulation of strings theory. Indeed, by construction [12, perturbative string theory is based on the well defined nature of scattering amplitudes of various excitations and hence fundamentally relies on the possibility to correctly define the $\mathcal{S}$ - matrix [13]. As such, formulating string theory in presence of a finite cosmic horizon is challenging. The so called Liouville string framework [14] represents an attempt to solve the problem in the case of non conformal backgrounds (including the de Sitter spacetime), but much work is still needed before a complete mathematical formulation is achieved.

A radically different approach is, however, possible. Indeed, observations do tell us that the universe is spatially flat and accelerating, but this by no way implies that the acceleration should be eternal. This latter feature turns out only as a consequence of assuming a positive $\Lambda$ term $\S$ to explain cosmic speed up. From an observational point of view, however, nothing prevents us to consider an effective dark energy fluid as a source of the accelerated expansion. We can then split its energy density as the sum of two components and then investigate the consequences on the future evolution of the universe. Such an approach has been recently investigated by some authors [15, 16, 17] with interesting results. In particular, in [16], some of us have presented a model whose $\S$ A similar argument also holds for a quintessence scalar field whatever is the self interaction potential. 
dark energy component is the sum of a negative cosmological constant and a quintessence scalar field evolving under the action of an exponential potential. As a result, although the model is presently accelerating, eternal acceleration disappears and the universe ends in a Big Crunch like singularity in a finite time. Motivated by these theoretical virtues, we further explore this model by the observational point of view in order to see whether a negative $\Lambda$ is indeed compatible with the astrophysical data at hand.

Notwithstanding the positive evidence of the model, it is also worth wondering whether an alternative theoretical derivation is possible. Actually, there are difficulties to reconcile a negative $\Lambda$ term within the framework of Quantum Field Theory. In the context of the Standard Model of Elementary Particle Physics, Spontaneous Symmetry Breaking induces a large (negative) value of the cosmological constant:

$$
\Lambda_{i n d}=-\frac{M_{H}^{2}}{8 \sqrt{2} G_{F}}
$$

If we apply the current experimental bounds on the mass of the Higgs boson $M_{H}$ and on the Fermi constant $G_{F}$, we obtain a (negative) value 55 orders of magnitude larger than the observed upper bound of the cosmological constant CC. There have been several attempts to solve this problem, without enough success [18]. A vacuum cosmological term $\Lambda_{v}$ with (positive) opposite sign is often introduced, so that the physical observable cosmological constant results :

$$
\Lambda_{p h}=\Lambda_{i n d}+\Lambda_{v}
$$

The introduction of $\Lambda_{v}$ is also dictated by the requirement of renormalizability of the massive theory, so the $\mathrm{CC}$ problem is rather the need of the extremely precise choice of the corresponding normalization condition $\Lambda_{p h}=0$ in the very far infrared. It is very difficult to explain why the two terms on the right hand side of above equation should cancel each other with such accuracy at that point. On the other hand, as for all quintessence models, it is not totally clear where the scalar field comes from. Having such a large dominance by dark ingredients is, for sure, a disturbing situation so that it is worth to look for other potential explanations.

Following the prescriptions of the Occam razor, it is desirable to have as less components of unknown origin as possible in a cosmological model. In order to overcome this problem, we resort to fourth order theories of gravity (also referred to in the following as $f(R)$ theories). According to this approach, cosmic acceleration is the first signal of a breakdown of General Relativity so that the gravity Lagrangian has to be modified by replacing the scalar curvature $R$ with a generic analytic function $f(R)[19,20,21,22$. Moreover, it has been shown [23] that it is always possible to find out a $f(R)$ theory reproducing the same dynamics (i.e., scale factor and Hubble parameter) as a given dark energy model. Using this method, we can reformulate the model above in terms of fourth order theories of gravity. The resulting $f(R)$ will allow to both fit the data and solve the problem of eternal acceleration without resorting to a controversial negative $\Lambda$ and an ad hoc scalar field. 
The plan of the paper is as follows. We present the starting cosmological model in Sect. 2 where we generalize the approach in [16] including the radiation term. The numerical solution for the expansion rate for this model is the key ingredient for the likelihood analysis discussed in Sect. 3 where we contrast the model against a wide set of observational data thus being able to constrain its characteristic parameters. Although our model comprises two ingredients other than standard matter and radiation, it is worth stressing that it is dynamically equivalent to a model dominated by a single dark energy fluid whose equation of state is reconstructed in Sect. 4. The best fit model is, on the other hand, used as input for the procedure of reconstructing $f(R)$ which is presented in Sect. 5 where we show that a negative $\Lambda$ could result as part of an effective representation of a fourth order theory. Sect. 6 is finally devoted to conclusions.

\section{The model}

Looking at the impressive amount of papers addressing the problem of cosmic acceleration clearly shows that two leading candidates to the dark energy throne are the old cosmological consntat $\Lambda$ and a scalar field $\phi$ evolving under the influence of its self-interaction potential $V(\phi)$. While a $\Lambda$ term adds a constant energy density $\rho_{\Lambda}$ and a negative pressure $p_{\Lambda}=-\rho_{\text {Lambda }}$ into the cosmic evolution equations, a scalar field partecipates to the dynamics through its energy density and pressure given as:

$$
\left\{\begin{array}{l}
\rho_{\phi}=\frac{1}{2} \dot{\phi}^{2}+V(\phi) \\
p_{\phi}=\frac{1}{2} \dot{\phi}^{2}-V(\phi)
\end{array}\right.
$$

with a dot denoting the derivative with respect to the cosmic time $t$. The evolution of the scalar field is then governed by its Klein-Gordon equation:

$$
\ddot{\phi}+3 H \dot{\phi}+\frac{d V}{d \phi}=0
$$

with $H=\dot{a} / a$ the Hubble parameter and $a$ the scale factor (normalized to unity at the present time). It is easy to show that Eq.(15) is the same as the continuity equation for the scalar field energy density $\rho_{\phi}$ under the hypothesis that $\phi$ does not interact with the matter and the other ingredients of the cosmic pie.

In the usual approach, one adds either a scalar field or a cosmological constant term to the field equations. However, since what we see is only the final effect of the dark energy components, in principle nothing prevents us to add more than one single component provided that the effective dark energy fluid coming out is able to explain the data at hand. Moreover, as we have hinted upon above, a single scalar field, while

explaining cosmic speed up, leads to a problematic eternal acceleration. A possible way out of this problem has been proposed by some of us [16] through the introduction of a negative cosmological term. Motivated by those encouraging results, we therefore 
consider a spatially flat universe filled by dust matter, radiation, scalar field and a (negative) cosmological constant term. The Friedmann equations thus read:

$$
\begin{aligned}
& H^{2}=\frac{1}{3}\left[\rho_{M}+\rho_{r}+\rho_{\Lambda}+\frac{1}{2} \dot{\phi}^{2}+V(\phi)\right], \\
& 2 \dot{H}+3 H^{2}=-\left[\frac{1}{3} \rho_{r}-\rho_{\Lambda}+\frac{1}{2} \dot{\phi}^{2}-V(\phi)\right],
\end{aligned}
$$

where we have used natural units with $8 \pi G=c=1$. Using the continuity equation for $\rho_{M}$ and $\rho_{r}$ and the definition of density parameter for the $\mathrm{i}$ - th fluid

$$
\Omega_{i}=\frac{\rho_{i}(a=1)}{\rho_{c}}=\frac{\rho_{i}(a=1)}{3 H_{0}^{2}},
$$

Eqs.(6) and (17) may be rewritten as:

$$
\begin{aligned}
& E^{2}=\Omega_{M} a^{-3}+\Omega_{r} a^{-4}+\frac{1}{2 H_{0}^{2}}\left(\frac{d \tilde{\phi}}{d t}\right)^{2}+\tilde{V}(\tilde{\phi}) \\
& H_{0}^{-1} \dot{E}=-\frac{3}{2} \Omega_{M} a^{-3}-2 \Omega_{r} a^{-4}-\frac{1}{2 H_{0}^{2}}\left(\frac{d \tilde{\phi}}{d t}\right)^{2}
\end{aligned}
$$

with $E=H / H_{0}, \tilde{\phi}=\phi / \sqrt{3}, \tilde{V}=V / 3 H_{0}^{2}$, and we denote with a subscript 0 the present day value of a quantity. It is convenient to introduce the dimensionless variable

$$
u=\ln (1+z)=-\ln a
$$

with $z=1 / a-1$ the redshift (having set $a_{0}=1$ ). In terms of this variable, the Friedmann equations become:

$$
\begin{aligned}
& E^{2}=\frac{\Omega_{M} \mathrm{e}^{3 u}+\Omega_{r} \mathrm{e}^{4 u}+\Omega_{\Lambda}+\tilde{V}(\tilde{\phi})}{1-(1 / 2)(d \tilde{\phi} / d u)^{2}}, \\
& \frac{1}{2} \frac{d E^{2}}{d u}=\frac{3}{2} \Omega_{M} \mathrm{e}^{3 u}+2 \Omega_{r} \mathrm{e}^{4 u}+\frac{3}{2} E^{2}\left(\frac{d \tilde{\phi}}{d u}\right)^{2},
\end{aligned}
$$

while the Klein-Gordon equation reads :

$$
E^{2} \frac{d^{2} \tilde{\phi}}{d u^{2}}+\left(\frac{1}{2} \frac{d E^{2}}{d u}-3 E^{2}\right) \frac{d \tilde{\phi}}{d u}+\frac{d \tilde{V}}{d \tilde{\phi}}=0 .
$$

As well known, given an expression for the scalar field potential $\tilde{V}(\tilde{\phi})$, only two out of the three equations (10), (11), (12) are independent. It is convenient to insert Eqs.(10) and (11) into Eq.(12) to get a single equation governing the evolution of the scalar field:

$$
E^{2} \frac{d^{2} \tilde{\phi}}{d u^{2}}+\left[\frac{3}{2} \Omega_{M} \mathrm{e}^{3 u}+\Omega_{r} \mathrm{e}^{4 u}+3 \Omega_{\Lambda}+3 \tilde{V}(\tilde{\phi})\right] \frac{d \tilde{\phi}}{d u}=0
$$


with $E^{2}(u)$ given by Eq.(10). In order to solve (numerically) this equation thus determining $\tilde{\phi}(u)$ and then $\mathrm{E}(\mathrm{u})$ through Eq.(10), one has to set two initial conditions. The first one can be trivially obtained by evaluating Eq.(10) in $u=0$ to get:

$$
\tilde{V}_{0}=\Omega_{\phi}-\frac{1}{2}\left(\frac{d \tilde{\phi}}{d u}\right)_{u=0}^{2}
$$

with $\tilde{V}_{0}=\tilde{V}\left(\tilde{\phi}_{0}\right)$. Given the shape of the potential $V(\phi)$, the above relation can be inverted to get the present day value of the scalar field. A second initial condition can be obtained by first considering the equation of state (hereafter EoS) of the field given as :

$$
w_{\phi}=\frac{p_{\phi}}{\rho_{\phi}}=\frac{E^{2}(d \tilde{\phi} / d u)^{2}-2 \tilde{V}}{E^{2}(d \tilde{\phi} / d u)^{2}+2 \tilde{V}} .
$$

Evaluating this at the present day and using the above relation for $\tilde{\phi}_{0}$, we finally get the initial conditions:

$$
\begin{aligned}
& \tilde{V}_{0}=\frac{\Omega_{\phi}}{2}\left(1+w_{0}\right), \\
& \left(\frac{d \tilde{\phi}}{d u}\right)_{u=0}^{2}=\Omega_{\phi}\left(1-w_{0}\right)
\end{aligned}
$$

with $w_{0}=w_{\phi}(u=0)$. Note that, since the left hand side of Eqs.(15) and (16) are positive definite, we get the constraint $-1 \leq w_{0} \leq 1$ which is always verified for any ordinary scalar field whatever the potential $\tilde{V}(\tilde{\phi})$ is.

\subsection{The deceleration parameter}

In order to separate models in accelerating and decelerating ones, one has to compute the present day value of the deceleration parameter defined as:

$$
q(u)=-\frac{\ddot{a} a}{\dot{a}^{2}}=-1-\frac{\dot{H}}{H^{2}}=-1+\frac{1}{E} \frac{d E}{d u} .
$$

Using Eqs.(10) and (11), we can therefore write the deceleration parameter as :

$$
\begin{aligned}
q=-1 & +\left[1-\left(\frac{d \tilde{\phi}}{d u}\right)^{2}\right] \\
& \times \frac{(3 / 2) \Omega_{M} \mathrm{e}^{3 u}+2 \Omega_{r} \mathrm{e}^{4 u}+(3 / 2)(d \tilde{\phi} / d u)^{2}}{\Omega_{M} \mathrm{e}^{3 u}+\Omega_{r} \mathrm{e}^{4 u}+\Omega_{\Lambda}+\tilde{V}(\tilde{\phi})} .
\end{aligned}
$$

Evaluating this relation in $u=0$ and using Eq.(16), the present day value then reads :

$$
q_{0}=\frac{1}{2}\left(1+\Omega_{r}\right)-\frac{3}{2}\left(\Omega_{\Lambda}+\Omega_{\phi} w_{0}\right)
$$


so that, in order to have accelerating models $\left(q_{0} \leq 0\right)$, we have to set the constraint:

$$
w_{0} \leq \frac{\Omega_{\Lambda}-\left(1+\Omega_{r}\right) / 2}{\Omega_{\phi}} .
$$

We can also solve Eq.(18) with respect to $w_{0}$ to get:

$$
w_{0}=-\frac{\left(1-2 q_{0}\right)+\Omega_{r}-3 \Omega_{\Lambda}}{3 \Omega_{\phi}}
$$

so that the initial conditions now rewrite:

$$
\begin{aligned}
& \tilde{V}_{0}=\left(3 \Omega_{\phi}+\Omega_{r}-3 \Omega_{\Lambda}+1-2 q_{0}\right) / 6, \\
& \left(\frac{d \tilde{\phi}}{d u}\right)_{u=0}^{2}=3 \Omega_{\phi}-\Omega_{r}+3 \Omega_{\Lambda}-1+2 q_{0} .
\end{aligned}
$$

Since the lhs of these relations are positive quantities, we get the following constraints on the present day deceleration parameter :

$$
\frac{1}{2}\left(1+\Omega_{r}\right)+\frac{3}{2}\left(\Omega_{\phi}+\Omega_{\Lambda}\right) \leq q_{0} \leq \frac{1}{2}\left(1+\Omega_{r}\right)+\frac{3}{2}\left(\Omega_{\phi}-\Omega_{\Lambda}\right) .
$$

For accelerating models, the lower limit in Eq.(23) must be negative so that we get :

$$
\Omega_{\Lambda} \leq \frac{1}{3}\left(1+\Omega_{r}\right)-\Omega_{\phi}
$$

Note that for models with a negative cosmological constant this relation is always satisfied thus meaning that for such a choice it is possible to work out accelerating solutions of the field equations.

\subsection{The exponential potential}

The above discussion holds whatever is the scalar field potential $V(\phi)$, but in order to actually solve the cosmic equations we have to definitively assign an analytical expression for this quantity. Following [24, 25], we consider an exponential potential:

$$
V(\phi)=B^{2} \exp (-\sigma \phi)
$$

with $B^{2}$ a generic constant and $\sigma^{2}=12 \pi G / c^{2}=3 / 2$. As shown in [26], such a choice leads to a cosmological model (made out of the above scalar field and dust matter) in good agreement with a large set of astrophysical data.

Although being interesting on both theoretical and observational grounds, such a model is however affected by the problem of eternal acceleration. As can be easily understood, since the matter energy density decreases as $a^{-3}$ (with $a$ the scale factor normalized to be unity at the present day), the universe becomes soon scalar field dominated and the accelerating expansion never ends. In order to avoid this problem, Cardenas et al. [16] have added a third ingredient to the cosmic pie, namely a negative cosmological constant. The Nöther symmetry approach [27] makes it possible to find a convenient change of variables in such a way that exact solutions are found. 
Although dealing with an analytical expression is welcome, the result in [16] only holds if the radiation term is neglected. While this is not a problem when considering the late universe, introducing radiation drastically changes the structure of the cosmic equations so that the Nöther symmetry approach does not apply anymore. As a consequence, the analytical solution may no more be used and a numerical analysis is needed. To this end, we insert Eq.(25) into Eq.(15) and solve with respect to $\tilde{\phi}_{0}$ :

$$
\tilde{\phi}_{0}=-\sqrt{\frac{2}{9}} \ln \left[\frac{3 H_{0}^{2}}{2 B^{2}} \Omega_{\phi}\left(1-w_{0}\right)\right]=-\sqrt{\frac{2}{9}} \ln \left[\Omega_{\phi}\left(1-w_{0}\right)\right]
$$

where, in the second equality, we have arbitrarily set $B^{2}=(3 / 2) H_{0}^{2}$. Note that such a choice has no effect on the dynamics since it is a simple rescaling of the scalar field $\tilde{\phi}$ which does not influence any physically interesting quantity. With such a choice, the potential and its derivative then read:

$$
\left\{\begin{aligned}
\tilde{V}(\tilde{\phi}) & =\frac{1}{2} \exp \left(-\frac{3}{\sqrt{2}} \tilde{\phi}\right) \\
\frac{d \tilde{V}}{d \tilde{\phi}} & =\frac{3}{2 \sqrt{2}} \exp \left(-\frac{3}{\sqrt{2}} \tilde{\phi}\right) .
\end{aligned}\right.
$$

Summarising, in order to determine the cosmic dynamics (i.e., the scale factor and the Hubble parameter) for such a model with both a negative cosmological constant $\Lambda$ and a scalar field $\phi$ with an exponential potential added to the standard dust matter and radiation terms, we have to numerically integrate Eq.(13) with the potential given by Eq.(27) and the initial conditions (26) and (16). The solution for $\tilde{\phi}(u)$ thus obtained may then be inserted into the potential and then into Eq.(10) to determine the Hubble parameter which can be further integrated to get the scale factor. In order to perform such a scheme, one has to set the values of up to four parameters, namely the dust and radiation density parameters $\Omega_{M}$ and $\Omega_{r}$, and the present day values $\left(\Omega_{\phi}, w_{0}\right)$ of the

scalar field energu density and EoS respectively. In particular, one could use Eq.(19) to choose $w_{0}$ in such a way that the resulting model will be today accelerating.

\section{Matching with the data}

Notwithstanding how well motivated it is, a whatever model must be able to reproduce what is observed. This is particularly true for the model we are considering because the presence of a negative cosmological constant introduces a positive pressure term potentially inhibiting the cosmic speed up. Moreover, contrasting the model against the data offers also the possibility to constrain its characteristic parameters and estimate other derived interesting quantities, such as $q_{0}$, the transition redshift $z_{T}$ and the age of the universe $t_{0}$. Motivated by these considerations, we will therefore fit our model to the dataset described below parametrizing the model itself with the matter density $\Omega_{M}$, the scalar field quantities $\left(\Omega_{\phi}, w_{0}\right)$ and the dimensionless Hubble constant $h$ (i.e., 
$H_{0}$ in units of $100 \mathrm{~km} / \mathrm{s} / \mathrm{Mpc}$ ), while we will set the radiation density parameter as $\Omega_{r}=10^{-4.3}$ as in [28] from a median of different values reported in literature.

\subsection{The method and the data}

In order to constrain the model parameters, we maximize the following likelihood:

$$
\mathcal{L} \propto \exp \left[-\frac{\chi^{2}(\mathbf{p})}{2}\right]
$$

where $\mathbf{p}=\left(\Omega_{M}, \Omega_{\phi}, w_{0}, h\right)$ denotes the set of model parameters and the pseudo- $\chi^{2}$ merit function reads:

$$
\begin{aligned}
\chi^{2}(\mathbf{p}) & =\sum_{i=1}^{N}\left[\frac{\mu^{t h}\left(z_{i}, \mathbf{p}\right)-\mu_{i}^{o b s}}{\sigma_{i}}\right]^{2}+\sum_{i=1}^{N}\left[\frac{f_{g a s}^{t h}\left(z_{i}, \mathbf{p}\right)-f_{g a s, i}^{o b s}}{\sigma_{i}}\right]^{2} \\
& +\left[\frac{\mathcal{A}(\mathbf{p})-0.474}{0.017}\right]^{2}+\left[\frac{\mathcal{R}(\mathbf{p})-1.70}{0.03}\right]^{2}+\left(\frac{h-0.72}{0.08}\right)^{2} .
\end{aligned}
$$

Let us discuss briefly the different terms entering Eq.(29). In the first one, we consider the distance modulus $\mu=m-M$, i.e. the difference between the apparent and absolute magnitude of an object at redshift $z$. This is given as:

$$
\mu(z)=m-M=25+5 \log D_{L}(z)
$$

with $D_{L}(z)$ the luminosity distance (in Mpc) defined as:

$$
D_{L}(z)=\frac{c}{H_{0}}(1+z) \int_{0}^{z} \frac{d z^{\prime}}{E\left(z^{\prime}, \mathbf{p}\right)} .
$$

As input data, we use the SNeIa sample assembled in [29] by putting on a common scale the data recently released from the SNLS [30] and ESSENCE collaborations [31] and the higher redshift SNeIa observed with HST in the GOODS survey [32]. As well known, the SNeIa Hubble diagram is unable to determine the Hubble constant $H_{0}$ since this quantity is degenerate with the (unknown) absolute magnitude $M$. As such, Davis et al. have put all the SNeIa on the same distance scale using $h=0.656$. In our analysis, however, we will leave $h$ as a free parameter so that Eq.(301) must be rewritten as:

$$
\mu(z)=25+5 \log \left(\frac{c}{H_{0}^{f i d}}\right)+5 \log \left(\frac{h^{f i d}}{h}\right)+5 \log d_{L}(z)
$$

with $H_{0}^{f i d}=100 h^{f i d}=65.6 \mathrm{~km} / \mathrm{s} / \mathrm{Mpc}$ and $d_{L}=D_{L} /\left(c / H_{0}\right)$ the Hubble free luminosity distance. Note that the value of $h$ determined by this fit will not be fully reliable because of the degeneracy hinted above. However, such a problem will not affect the estimates of the other parameters since we will marginalize over $h$ in the analysis of the results.

The second term in Eq.(29) relies on the gas mass fraction in galaxy clusters. We briefly outline here the method referring the interested reader to the literature for further details [33, 34, 35]. Both theoretical arguments and numerical simulations predict that 
the baryonic mass fraction in the largest relaxed galaxy clusters should be invariant with the redshift (see, e.g., Ref. [36]). However, this will only appear to be the case when the reference cosmology in making the baryonic mass fraction measurements matches the true underlying cosmology. From the observational point of view, it is worth noting that the baryonic content in galaxy clusters is dominated by the hot X-ray emitting intra-cluster gas so that what is actually measured is the gas mass fraction $f_{\text {gas }}$ and it is this quantity that should be invariant with the redshift within the caveat quoted above. Moreover, it is expected that the baryonic fraction in clusters equals the universal ratio $\Omega_{b} / \Omega_{M}$ so that $f_{\text {gas }}$ should indeed be given by $b \times \Omega_{b} / \Omega_{M}$ where the multiplicative factor $b$ is motivated by simulations that suggest that the gas fraction is slightly lower than the universal ratio because of processes that convert part of the gas into stars or eject it outside the cluster.

Following Ref. [37], we adopt the concordance $\Lambda$ CDM model (i.e., with $\Omega_{M}=0.3$, $\left.\Omega_{\Lambda}=0.7, h=0.7\right)$ as reference cosmology in making the measurements so that the theoretical expectation for the apparent variation of $f_{\text {gas }}$ with the redshift is [37]:

$$
f_{\text {gas }}(z)=\frac{K \gamma A(z) b(z)}{1+s(z)} \frac{\Omega_{b}}{\Omega_{M}}\left[\frac{D_{A}^{\Lambda C D M}(z)}{D_{A}(z, \mathbf{p})}\right]^{3 / 2} .
$$

Some words are needed to explain the meaning of the different terms entering the above equations. First, the two functions $b(z)$ and $s(z)$ take into account variations of the ratio $\Omega_{b} / \Omega_{M}$ due to gastrophysics and star formation respectively. In a first approximation,. they are described as linear functions of the redshift. However, in order to not increase the number of parameters, we will set them to constant values taking $b(z)=b_{0}=0.83$ and $s(z)=s_{0}=0.16(h / 0.70)^{1 / 2}$ in agreement with [37]. While $K$ and $\gamma$ are normalizing factors correcting for measurement related problems and can be put to the constant values 1.0 and 1.05 respectively, $A(z)$ actually plays a more important role. To understantd its origin, it is worth remembering that $f_{\text {gas }}$ is typically measured at a given fraction of the cluster virial radius. However, on the sky distances are measured in angular rather than physical units with the conversion depending on the assumed cosmological model. Since the reference model is different from the actual one, a correction term must be included to account for the change in the radius. Considering the scaling of the different quantities involved in the measurement process, it is possible to show that the correction term may be approximated as [37]:

$$
A(z)=\left[\frac{E(z) D_{A}(z, \mathbf{p})}{E^{\Lambda C D M}(z) D_{A}^{\Lambda C D M}(z)}\right]^{\eta}
$$

with $E^{\Lambda C D M}(z)=\left[\Omega_{M}(1+z)^{3}+\Omega_{\Lambda}\right]^{1 / 2}$ and $\eta=0.214$. Note that, actually, because of the small $\eta$ value, this term does not play a significant role in the fitting process, while a key ingredient is the last one in Eq.(33) given by the ratio of the angular diameter distances $D_{A}(z)=D_{L}(z) /(1+z)^{2}$ between the $\Lambda \mathrm{CDM}$ and the model to be tested. It is worth noting that $f_{\text {gas }}(z)$ depends not only on the integrated Hubble parameter, but also explicitly on the baryon and total matter density parameters $\Omega_{b}$ and $\Omega_{M}$. In particular, 
baryogenesis calculations contrasted to the observed abundances of primordial elements puts a severe constraint on the physical baryon density $\omega_{b}=\Omega_{b} h^{2}$. Using this method, Kirkman et al. [38] have determined:

$$
\omega_{b}=0.0214 \pm 0.0020
$$

A rigorous analysis should be done letting $\omega_{b}$ as a free parameter eventually including the above estimates as a prior. However, in order to not increase the number of parameters to be determined, we will set $\omega_{b}=0.0214$ neglecting the small error.

The third term in the definition of $\chi^{2}$ takes into account the measurement of the baryonic acoustic oscillation (BAO) peak in the large scale correlation function at $100 \mathrm{~h}^{-1} \mathrm{Mpc}$ separation detected by Eisenstein et al. 39] using a sample of 46748 luminous red galaxies (LRG) selected from the SDSS Main Sample [40. Actually, rather than the position of acoustic peak itself, a closely related quantity is better constrained from these data, namely the acoustic peak parameter defined as [39] :

$$
\mathcal{A}=\frac{\sqrt{\Omega_{M}}}{z_{L R G}}\left[\frac{z_{L R G}}{E\left(z_{L R G}\right)} y^{2}\left(z_{L R G}\right)\right]^{1 / 3}
$$

with $z_{L R G}=0.35$ the effective redshift of the LRG sample, and we have introduced the dimensionless coordinate distance $y(z)$ defined as:

$$
y(z)=\int_{0}^{z} \frac{d z^{\prime}}{E\left(z^{\prime}, \mathbf{p}\right)} .
$$

As it is clear, the $\mathcal{A}$ parameter depends not only on the dimensionless coordinate distance (and thus on the integrated expansion rate), but also on $\Omega_{M}$ and $E(z)$ explicitly which removes some of the degeneracies intrinsic in distance fitting methods. Therefore, it is particularly interesting to include $\mathcal{A}$ as a further constraint on the model parameters using its measured value [39] :

$$
\mathcal{A}=0.469\left(\frac{n_{s}}{0.98}\right)^{-0.35} \pm 0.017
$$

with $n_{s}$ the spectral index of the primordial density perturbations. For $n_{s}=0.95$ as determined by the WMAP 3rd year analysis [41, we get $\mathcal{A}=0.474 \pm 0.017$ as set in Eq.(29). A caveat is in order here. As discussed in [39], the measurement of the position of the BAO peak from the correlation function relies somewhat on having assumed the $\Lambda \mathrm{CDM}$ model to convert angular distances in physical distances and in the computation of the reference (smoothed) power spectrum. As a consequence, one should use with caution the above value for $\mathcal{A}$ when using a different cosmological model and rather directly fitting the measured correlation function. However, it is expected that the position of the BAO peak does not change too much in a different model since it is measured at a relatively low redshift. Since a full analysis will require the solution of the perturbation equations for our model (which is outside our aims here), we will follow the common practice in literature neglecting this problem and directly using the $\mathcal{A}$ parameter as an observational constraint included in the $\chi^{2}$ merit function. 
Let us consider the fourth term in Eq.(29) which relies on the shift parameter [42] :

$$
\mathcal{R}(\mathbf{p})=\sqrt{\Omega_{M}} y\left(z_{L S}, \mathbf{p}\right)
$$

with $z_{L S}$ the redshift of the last scattering surface which we compute using the approximation given in [43]. Using the WMAP3 data, Wang \& Mukherjee [44] have determined $\mathcal{R}=1.70 \pm 0.03$ in very good agreement with what is expected for the concordance $\Lambda \mathrm{CDM}$ model. Note that such a result may argue in favour of models with a negligible dark energy component at high redshift, but we defer this discussion to the later analysis of the results.

Finally, the term depending on $h$ in Eq.(29) is only a Gaussian prior on this quantity obtained by considering the model independent estimate of the Hubble constant recovered by the HST Key project [45]. This collaboration have measured $H_{0}$ using a wide set of different local distance calibrators thus ending up with a value which is claimed to fully take into account any possible systematic error inherent to the peculiarities of each single method. Comfortably, such a measurement turns out to be in good agreement with other (less precise) methods relying on different physics and distance scales as the time delay in lensed quasars [46] and the Sunyaev-Zel'dovich effect [4] in galaxy clusters.

In order to maximize the likelihood function $\mathcal{L}(\mathbf{p})$, we should compute it over a very fine grid in the four dimensional parameter space $\left(\Omega_{M}, \Omega_{\phi}, w_{0}, h\right)$ and then interpolate the results for values falling in between two grid points. This is quite time consuming for a very fine grid so that we resort to a Monte Carlo Markov Chain (MCMC) method running three chains with 30000 points each and assessing the convergence using the Gelman-Rubin test (with $|R-1|=0.1$ ). It is worth stressing that, in order to be sure that the MCMC runs into the region with negative $\Lambda$, it is better to reparametrize the model using $\Omega_{\Lambda}$ instead of $\Omega_{\phi}$ as model parameter using the obvious relation:

$$
\Omega_{\phi}=1-\Omega_{M}-\Omega_{r}-\Omega_{\Lambda} .
$$

Therefore, in the following, we will use $\left(\Omega_{M}, \Omega_{\Lambda}, w_{0}, h\right)$ as the parameter space to be explored by the MCMC code. After cutting the burn - in period, the final coadded chain contains $\simeq 88000$ points thus guaranteeing an efficient coverage of the interesting region of the parameter space allowing us to compute the marginalized likelihood functions for each parameter $p_{i}$ :

$$
\mathcal{L}_{p_{i}}\left(p_{i}\right) \propto \int d p_{1} \ldots \int d p_{i-1} \int d p_{i+1} \ldots \int d p_{n} \mathcal{L}(\mathbf{p})
$$

which is then normalized at unity at maximum. Under the Bayesian framework, the best estimated for the parameter $p_{i}$ is given by the median of the marginalized likelihood, while the 68 and $95 \%$ confidence ranges are given as $\left(x_{1 \sigma}, y_{1 \sigma}\right)$ and $\left(x_{2 \sigma}, y_{2 \sigma}\right)$ with $x_{i \sigma}$ and $y_{i \sigma}$ computed by solving respectively the equations:

$$
\int_{p_{i, \text { min }}}^{x_{i \sigma}} \mathcal{L}_{p_{i}}\left(p_{i}\right) d p_{i}=\delta_{i} \int_{p_{i, \text { min }}}^{p_{i, \max }} \mathcal{L}_{p_{i}}\left(p_{i}\right) d p_{i},
$$


Table 1. Best fit $(b f)$ and median (med) values and $1 \sigma$ and $2 \sigma$ ranges of the parameters $\left(\Omega_{M}, \Omega_{\Lambda}, w_{0}, h, \Omega_{\phi}\right)$ as obtained from the likelihood analysis.

\begin{tabular}{|c|c|c|c|c|}
\hline Par & $b f$ & med & $1 \sigma$ & $2 \sigma$ \\
\hline$\Omega_{M}$ & 0.283 & 0.307 & $(0.272,0.352)$ & $(0.246,0.410)$ \\
$\Omega_{\Lambda}$ & -0.072 & -0.298 & $(-0.54,-0.11)$ & $(-0.92,-0.02)$ \\
$w_{0}$ & -0.72 & -0.67 & $(-0.74,-0.60)$ & $(-0.79,-0.53)$ \\
$h$ & 0.632 & 0.620 & $(0.588,0.654)$ & $(0.554,0.692)$ \\
$\Omega_{\phi}$ & 0.789 & 0.989 & $(0.799,1.226)$ & $(0.700,1.574)$ \\
\hline
\end{tabular}

$$
\int_{y_{i \sigma}}^{p_{i, \max }} \mathcal{L}_{p_{i}}\left(p_{i}\right) d p_{i}=\delta_{i} \int_{p_{i, \min }}^{p_{i, \max }} \mathcal{L}_{p_{i}}\left(p_{i}\right) d p_{i},
$$

with $\delta_{i}=(1-0.68) / 2$ for $i=1$ and $(1-0.95) / 2$ for $i=2$ and $\left(p_{i, \min }, p_{i, \max }\right)$ the lower and upper bounds chosen for the parameter $p_{i}$. For the model we are considering, we conservatively let $\Omega_{M}$ range between 0.15 and 0.45 , while the range for $h$ is $(0.45,0.85)$. While the upper bound for $\Omega_{\Lambda}$ is dictated by our constraint $\Lambda<0$, choosing an upper limit is a more complicated issue. Indeed, the scalar field and the negative cosmological constant may be incorporated in a single dark energy fluid (as we will see later) so that there is a degeneracy in balancing the two individual components. We therefore arbitrarily set $\Omega_{\Lambda}>-1.5$ thus allowing values of $\Omega_{\phi}$ quite larger than 1 . Finally, we cut the physically acceptable range $(-1,1)$ for $w_{0}$ to become $(-1,0)$ which is still a conservative choice. Indeed, we could also use Eq.(19) to set an upper bound for $w_{0}$ ensuring $q_{0} \leq 0$. However, we prefer to be fully open minded not forcing the chains to explore a priori acccelerating models only.

\subsection{Results}

Best fit model parameters, median values and 1 and $2 \sigma$ ranges for the parameters $\left(\Omega_{M}, \Omega_{\Lambda}, w_{0}, h, \Omega_{\phi}\right.$ are reported in Table 1, while Figs.1 and 2 shows how well our best fit model reproduce the data on the SNeIa Hubble diagram and gas mass fraction.

Let us first discuss some general points. First, we note that the best fit model is in quite good agreement with both the SNeIa and gas data. Indeed, the $\chi^{2}$ values are respectively 206 and 48 to be contrasted witth the number of datapoints, being 192 and 42 respectively. Less good, but still in satisfactory agreement with the observed ones, are the values for the acoustic peak and shift parameters being:

$$
\mathcal{A}=0.45, \quad \mathcal{R}=1.67 .
$$

Motivated by these results, we can therefore safely conclude that including a negative $\Lambda$ leads to a model still in agreement with the data so that this approach to halting eternal acceleration is a viable one from an observational point of view. In a sense, this is not surprising given that the net effect of the scalar field and negative $\Lambda$ is to provide a dark energy fluid with negative pressure dominating the energy budget. Neveretheless, it is 


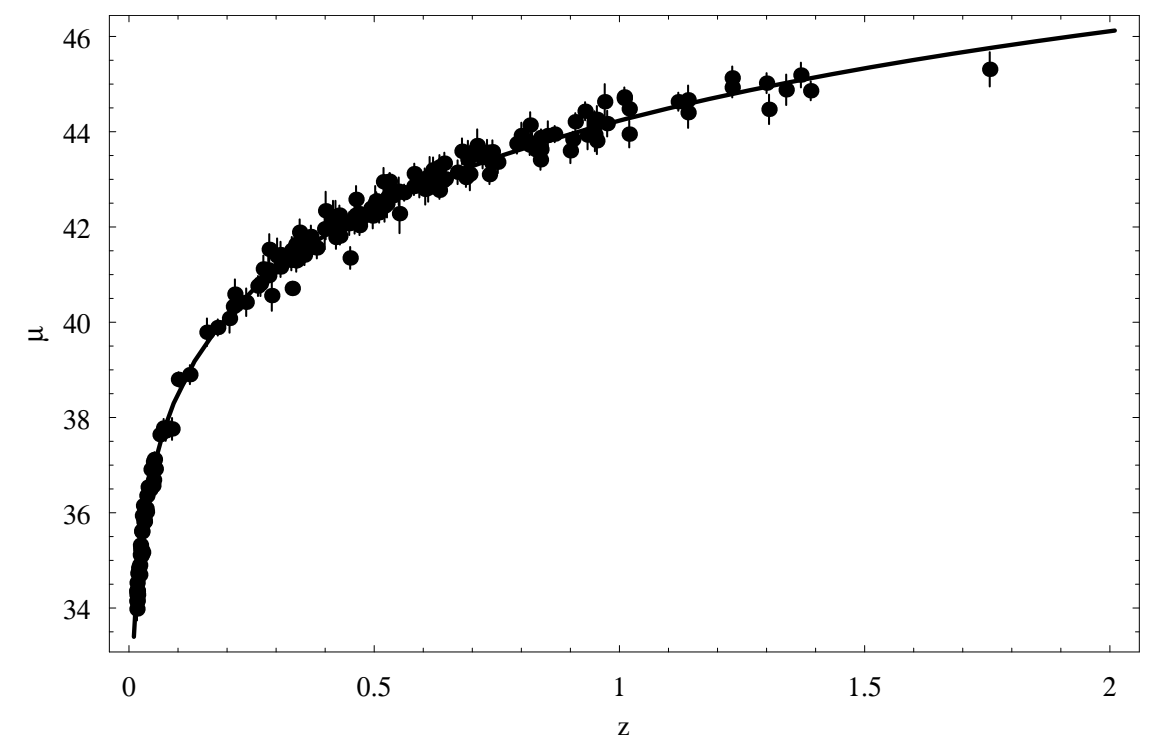

Figure 1. Best fit curve superimposed to the data on the SNeIa Hubble diagram.

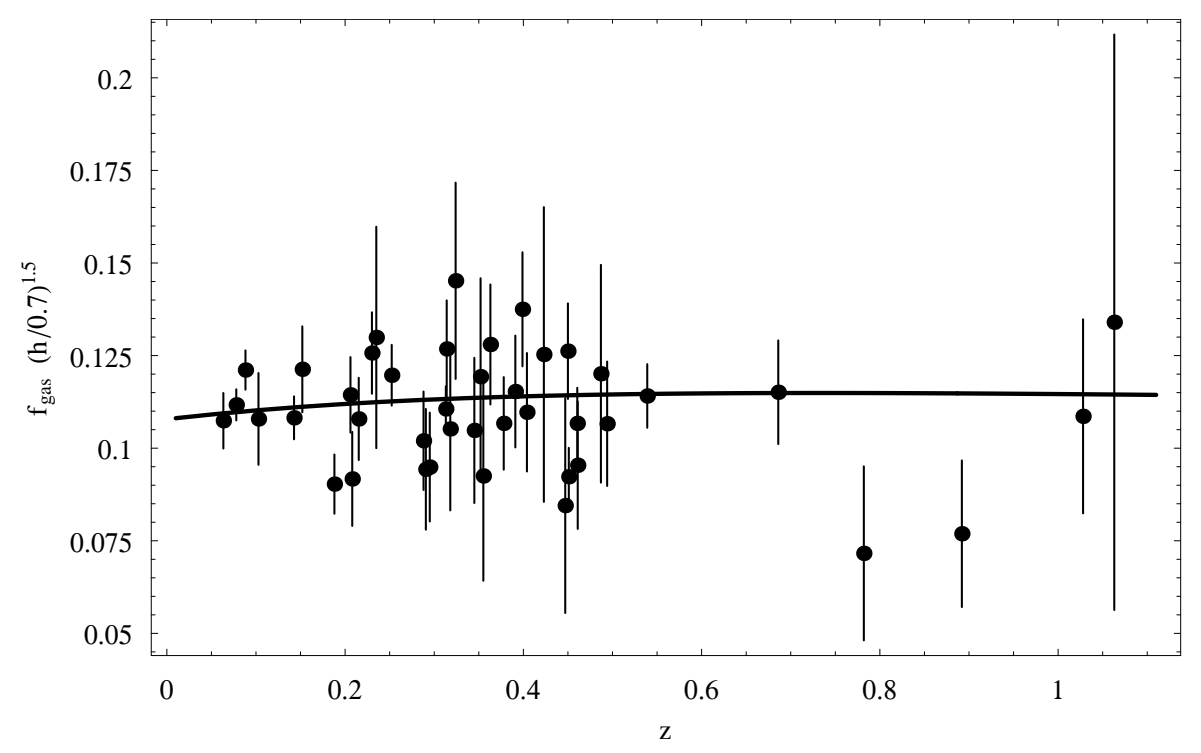

Figure 2. Best fit curve superimposed to the data on the gas mass fraction. Note that the theoretical curve plots indeed $f_{\text {gas }}(z) \times(h / 0.7)^{1.5}$ with $h$ set to its best fit value.

worth stressing that, while the one fluid model has to resort to a peculiar EoS, the two fluids scenario relies on quite simple ingredients such as the exponential potential and well motivated cosmological constant.

Comparing the best fit and median values of each parameter, it is soon clear that they are well different. This is, however, not a peculiarity of the model or the analysis, but rather a typical consequence of the non Gaussian shape of the likelihood. Since the full likelihood $\mathcal{L}\left(\mathbf{p}\right.$ is not the product of the marginalized likelihoods $\mathcal{L}_{p_{i}}\left(p_{i}\right)$, the best fit values $\mathbf{p}_{b f}$ are not given by the set of parameters that maximize each single $\mathcal{L}_{p_{i}}\left(p_{i}\right)$. Moreover, the marginalized likelihoods are markedly asymmetric so that the median 
values may be quite different from the maximum values. This is particularly the case for $\Omega_{\Lambda}$ because of the constraint imposed by hand on the negativeness of $\Lambda$. It is worth stressing, however, that all these caveats are common to the Bayesian approach to every data fitting problem so that they have not to be considered as model shortcomings.

A successful fit to the data could, however, be meaningless if the estimated parameters take unrealistic values. It is therefore worth discussing how our constraints compare with other previous estimates. Let us first consider, therefore, the matter density parameter $\Omega_{M}$. It is remarkable that the values in Table I are larger than the WMAP3 best fit model [41, being $\Omega_{M}=0.268 \pm 0.018$. Taken at face values, the two results are markedly different, even if they well overlap at $1 \sigma$. It is worth stressing, however, that the WMAP3 result reported above also depends on the cosmological model fitted to the data. For instance, adding the running of the spectral index to the fiducial $\Lambda$ CDM scenario shifts upward the estimated $\Omega_{M}$ now giving $\Omega_{M}=0.282 \pm 0.020$ in better agreement with our constraint. Moreover, our estimate also overlaps well with $\Omega_{M}=0.27 \pm 0.04$ from fitting the Chevallier-Polarski-Linder (CPL) model $w(z)=w_{0}+w_{1} z /(1+z)$ [48 to a dataset similar to our own [29], but not inlcuding the gas data. As such, we are confident that the higher than usual matter content of our model is perfectly reasonable and do not consider this as a worrisome shortcoming. It is worth noting that a higher value for $\Omega_{M}$ is somewhat expected. Indeed, in order to compensate for the attractive effect of the negative cosmological constant, a higher than usual contribute from the scalar field is needed. But such a large $\Omega_{\phi}$ should drive the universe towards a kind of superacceleration that needs to be compensated for by a larger matter content. The balance among these contrasting effects then induces a pushes $\Omega_{M}$ towards larger values. As a final remark, we also note that the 1 and $2 \sigma$ ranges are quite larger, while typically $\Omega_{M}$ is better constrained in literature. On one hand, this is a consequence of the degeneracy hinted above among three density parameters $\left(\Omega_{M}, \Omega_{\Lambda}, \Omega_{\phi}\right)$ rather than the only two, $\left(\Omega_{M}, \Omega_{D E}\right)$, typically entering dark energy models. On the other hand, we are not using here either the CMBR spectrum which probes the very high redshift (where $\Omega_{M}$ is the dominant term) or the LSS data (which are sensitive to the matter content). It is therefore not surprising that the error we achieve on $\Omega_{M}$ is so large.

While it is meaningless to compare the value of $\Omega_{\Lambda}$ to the other estimates in literature given that we are considering a negative rather than positive $\Lambda$, one could naively think that $w_{0}$ should be compared to other determinations. However, it is easy to understand that this is not the case. Referring to the CPL parametrization as a prototype for phenomenological EoS and using a similar dataset as our own, Davis et al. 29] have obtained $w_{0}=-1.1_{-0.3}^{+0.4}$. Compared with the values in Table 1, we find only a marginal agreement at $2 \sigma$ level. However, the CPL parametrization assumes a single dark energy fluid, while our model comprises two different fluids so that the two results are not comparable. Qualitatively, the smaller is $w_{0}$ (in absolute value), the smaller is the driving force speeding up the universe expansion so that one could be surprised that our model fits so well the data. Actually, what really matters is not 
the EoS, but rather than the pressure $p$. Considering its present day value, we have $p_{\phi}(z=0)=w_{0} \Omega_{\phi} \rho_{\text {crit }}=-0.57 \rho_{\text {crit }}$ which compares qualitatively well to the CPL value $p_{C P L}=\left(1-\Omega_{M}\right) w_{0} \rho_{\text {crit }}=-0.37 \rho_{\text {crit }}$. Moreover, one should also take into account the different $h$ value which leads to different values of $\rho_{\text {crit }}$. Considering that the difference somewhat fades away when integrating the Hubble parameter to get the luminosity distance, it is therefore not surprising that the Hubble diagram is almost the same betweem the one fluid phenomenological CPL model and our two fluids scenario.

We do not discuss here the constraints on $h$ reported in Table 1 since, as we have yet said above, this quantity is degenerate with the SNeIa absolute magnitude. Note that $h$ does not enter either in the acoustic peak nor the shift parameters so that they do not help in breaking this degeneracy. On the other hand, the gas mass fraction $f_{\text {gas }}(z)$ weakly depends on $h$ since it enters the function $A(z)$ defined in (34) trhough $D_{A}(z)$. It is easy to check that $A(z) \propto\left(h / h_{\Lambda C D M}\right)^{-\eta}$ so that for $\eta=0.214$, we get indeed a negligible effect. With all these caveats in mind, we do not care much about our findings on $h$ which turns out to be lower than the $h \simeq 0.72$ preferred by the local measuremnts [45] and CMBR data [41]. Motivated by these considerations, we have therefore marginalized over $h$ in the discussion of the results, although it is worth stressing that the difference between $h$ and $h_{\Lambda C D M}=0.7$ has to be taken into account when comparing $h$ dependent quantities between the two models.

As a byproduct of the MCMC code, we can also estimate some interesting derived quantities to be contrasted again measurements in order to get some more tests on the viability of the model. To this aim, given a quantity $g(\mathbf{p})$, we compute its value for the points belonging to the MCMC and estimate its median value and 1 and $2 \sigma$ ranges as described above. In order to both speed up the computation and reduce the correlation between neighbouring points, we have thinned the chains extracting one over eight points thus ending up with a sample of $\simeq 11000$ values which is enough to get reliable estimates.

First, we discuss the results for the acoustic peak parameter whose median value and 1 and $2 \sigma$ ranges read:

$$
\mathcal{A}=0.460, \mathcal{A} \in(0.434,0.490), \mathcal{A} \in(0.407,0.523) \text {. }
$$

Pending the question about the validity of its measured value in models other than the $\Lambda \mathrm{CDM}$ one, we can therefore conclude that $\mathcal{A}$ predicted by our scenario is in excellent agreement with what is inferred from the correlation function of LRG galaxies. It is nevertheless worth extending this analysis by computing the power spectrum and hence the correlation function predicted by our model, although this is outside our aims here.

As a second interesting quantity, we consider the shift parameter for which we find:

$$
\mathcal{R}=1.38, \quad \mathcal{R} \in(1.21,1.53), \quad \mathcal{R} \in(1.06,1.65),
$$

these values being respectively the median and 1 and $2 \sigma$ ranges. A comparison with the measured value, $\mathcal{R}=1.70 \pm 0.03$, should make us conclude that the model is not able to fit this quantity. While this is somewhat expected on the basis of the equivalent 
dark energy model described in the next section, a more subtle issue has to be taken into account. Looking at Eq.(37) and considering the constraints on $\Omega_{M}$ in Table I, it is easy to conclude that the problem with $\mathcal{R}$ originates from $y\left(z_{L S}\right)<y_{\Lambda C D M}\left(z_{L S}\right)$. We have checked that this is indeed the case comparing $y(z)$ for our best fit model with the $\Lambda$ CDM curve. Actually, $y(z)$ deviates from $y_{\Lambda C D M}(z)$ more and more as $z$ increases. Although worrisome, the smaller value of $\mathcal{R}$ should not be considered as a strong motivation against our model. As explained in [49, 50], $\mathcal{R}$ is an approximation for the acoustic scale $\ell_{a}$ which is defined as:

$$
\ell_{a}=\frac{\pi\left(1+z_{L S}\right) D_{A}\left(z_{L S}\right)}{\int_{0}^{1+z_{L S}} c_{S} d a /(a \dot{a})}
$$

with $c_{s}$ the sound speed. The shift parameter is obtained by $\ell_{a}$ using a different normalization and approximating the denominator as $\Omega_{M}^{-1 / 2}$. This is correct only if the dark energy fades away with $z$ becoming subdominant at the last scattering. While this is true for the $\Lambda \mathrm{CDM}$ and standard quintessence models, this is not for our model as we will see in the next section. One should therefore compute $\ell_{a}$ for our case and compare with the estimated $\ell_{a}=303.6_{-1.2}^{+1.1}[50]$. Unfortunately, we are unable to perform such a computation here because the numerical solution for $\phi(z)$ and hence $a(t)$ becomes seriously unstable for large $z$. As a consequence, the integral in Eq.(39) turns out to be unreliable so that we can not discuss anymore this point leaving it for further work.

As a final issue, we also consider the age of the universe. Proceeding as before, we get (in Gyr) :

$$
t_{0}=13.9, \quad t_{0} \in(13.0,14.9), t_{0} \in(12.2,15.9),
$$

with the same meaning as before for the values reported. Compared with $t_{0}=$ $13.75 \pm 0.15$ Gyr estimated for the concordance $\Lambda$ CDM model from a combined analysis of the three-year WMAP, SNeIa and BAO data [41], our result is in very good agreement. It is nevertheless worth stressing that the WMAP3 constraint is strongly model dependent so that one should better compare with the model independent measurements. However, Krauss \& Chaboyer [51] have estimated $t_{0}=12.6_{-2.6}^{+3.4} \mathrm{Gyr}$ from globular clusters, while a similar value, $t_{0}=12.5 \pm 3.5 \mathrm{Gyr}$, has been determined from nucleochronology [52]. Given the larger error bars, it is not surprising that our estimate is in very good agreement with these values too thus giving a further strong evidence in favour of our scenario.

\section{Effective dark energy}

The model we are considering is made out of two non standard fluids, namely a negative cosmological constant and a scalar field. From a phenomenological point of view, however, we can work out a model comprising a single dark energy fluid giving rise to the same expansion rate as the present one. Ies EoS may be evaluated as:

$$
1+w_{e f f}(u)=\frac{(2 / 3) d \ln E(u) / d u-\left[\Omega_{M} \mathrm{e}^{3 u}+\frac{4}{3} \Omega_{r} \mathrm{e}^{4 u}\right] E^{-2}(u)}{1-\left(\Omega_{M} \mathrm{e}^{3 u}+\Omega_{r} \mathrm{e}^{4 u}\right) E^{-2}(u)}
$$




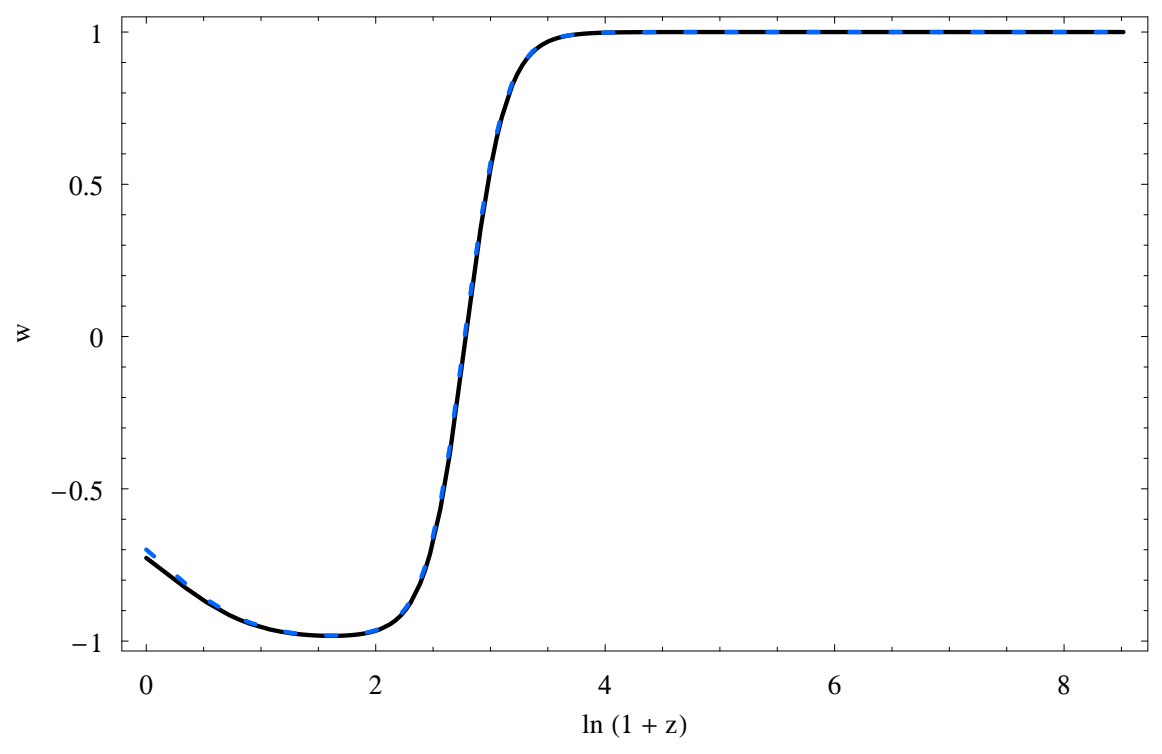

Figure 3. Scalar field (solid line) and effective dark energy (dashed line) EoS as function of $z$ for the best fit model.

so that the energy density may be then computed as:

$$
\rho_{D E}(z) / \rho_{c r i t}=\Omega_{D E} \exp \left\{3 \int_{0}^{z} \frac{1+w_{D E}\left(z^{\prime}\right)}{1+z^{\prime}} d z^{\prime}\right\} .
$$

Fig. [3 shows the reconstructed effective dark energy EoS for our model setting the parameters $\left(\Omega_{M}, \Omega_{\Lambda}, w_{0}\right)$ to the best fit value determined by the MCMC code. There are some interesting issues than can be drawn from this plot. First, we note that $w_{\text {eff }}(z)$ and $w_{\phi}(z)$ track each other being essentially equal $\amalg$ both in the late $(u<2)$ and early $(u>4)$ universe. This is not surprising since, for the best fit values we are using, the negative cosmological constant is quite small. However, a similar behaviour is obtained also for other values of the model parameters so that this is a rather common feature. As such, we will not investigate in detail how the shape of $w_{e f f}(z)$ depends on $\left(\Omega_{M}, \Omega_{\phi}, w_{0}\right)$, but our discussion below for the best fit model is nevertheless quite general.

As it is apparent, $w_{e f f}(z)$ cannot be fitted by the most common phenomenological EoS parametrization such as the CPL one. Indeed, while for the CPL ansatz $d w / d z=$ $(1+z)^{-2}$ has a monotonic shape, in our model $d w_{e f f} / d z$ is not at all monotonic and actually also changes its sign. For the best fit model shown in the plot, $w_{\text {eff }}(z)$ first decreases down to the almost $\Lambda \mathrm{CDM}$ value $w_{\text {eff }} \simeq-0.98$ for $z \simeq 4.0$ and then starts increasing crossing the dust value $w_{\text {eff }}=0$ at $z \simeq 15$ to finally stays constant to the stiff matter value $w_{\text {eff }}=1$ for $z \geq 210$. Varying $\left(\Omega_{M}, \Omega_{\Lambda}, w_{0}\right)$ changes these values, but not the shape of $w_{\text {eff }}$ which always asymptotes to 1 . Notwithstanding this unusual feature, the present day values of $w_{\text {eff }}$ and $d w_{\text {eff }} / d z$ are well within the $2 \sigma$ contours

$\|$ Actually, in Fig. 3, the two curves for $w_{\phi}(z)$ and $w_{\text {eff }}(z)$ are almost perfectly superimposed so that they can be hardly distinguished. However, we have checked that the larger in $\Omega_{\Lambda}$ (in abslute value), the larger is the difference between the two EoS. 


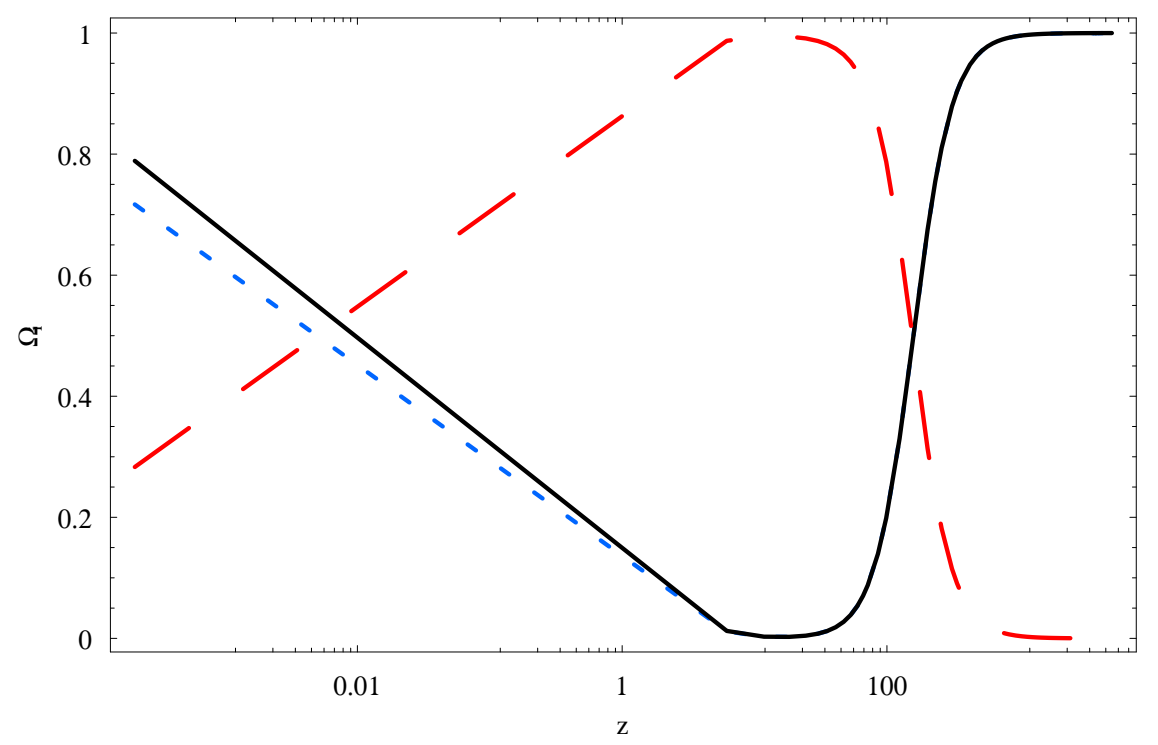

Figure 4. Effective dark energy (black solid), scalar field (blue short dashed) and matter (red long dashed) density parameters as function of $z$ for the best fit model.

one can obtain when fitting the CPL formula to the same dataset we have used here. This is not unexpected given that, for $z<1, D_{L}(z)$ for a linear EoS closely matches the luminosity distance-redshift relation predicted by our model.

As an alternative approach, one could resort to a model independent reconstruction of the EoS (see, e.g., [53] and references therein). Comparing $w_{\text {eff }}$ in Fig. 3 with Fig. 3 (right panel) in [53] shows us that the effective EoS significantly departs from what can be reconstructed by the data. It is nevertheless worth noting that in both cases the EoS asymptotes $w=1$ even if the transition is still more rapid in the case of the reconstructed EoS because of the lack of the initially decreasing phase we have in our model. Although troublesome, we are confident that the discrepancy between the reconstructed EoS and our $w_{e f f}(z)$ should not be considered as a strong evidence against the present model. Indeed, data do not tell anything on $w(z)$ directly, but only on the luminosity (or angular diameter) distance as function of the redshift. As such, one should not be worry about not reproducing a given $w(z)$, but the aim of the model must only be to fit the data with physically meaningful values of the model parameters. This task is successfully accomplished by our model so that we still retain it as a valid alternative to a single dark energy fluid scenario.

A potentially more worrisome problem is represented by the behaviour of the density parameter $\Omega_{D E}(z)$ when compared to the matter one $\Omega_{M}(z)$. As it is apparent from Fig.4, $\Omega_{D E}(z)$ closely follows $\Omega_{\phi}(z)$ being only slightly larger because of the need for compensating the negative cosmological consntat term. From this plot, it is clear that the usual sequence dark energy - matter - radiation dominions is not realized in this model since the matter era is followed by another dark energy dominated epoch. As such, the model could be troublesome when dealing with the nucleosynthesis which takes place during the radiation era. Indeed, it is usually claimed that, in order the BBN to 
be efficient, the constraints $\Omega_{D E}(1 \mathrm{Mev}) \leq 0.1$ has to be imposed which is strikingly violated by our effective dark energy model. It is, however, worth noting that such a constraint usually refers to a negative pressure fluid, while $w_{\text {eff }}$ is positive for our model. Exploring whether BBN can take place in such a background is outside our aim here, but we will return later on this topic in the conclusions.

While the BBN epoch is far away in a redshift range where our model could not be trusted anymore (for reasons explained later), structure formation typically probes a nearer epoch of the universe evolution. In the usual scenario, the growth of structure takes place during the matter dominated era well after the universe has left the radiation epoch. However, in our case, the radiation dominated era has been replaced by a dark energy dominated one and, moreover, the matter term is the leading one for a shorter than usual period. For instance, in the concordance $\Lambda \mathrm{CDM}$ scenario, matter drives universe expansion from $z \simeq z_{e q} \sim 3000$, while, for the best fit model in Fig. 4, the matter epoch starts later at $z \simeq 157$. Studying in detail the growth of structure and its impact on observable quantities such as the galaxy clusters mass function and abundance is outside our aims here and will be deeply investigated in a forthcoming publication. However, we can anticipate that this is a subtle issue. On the one hand, denoting with $\delta$ the matter density fluctuations, one usually has:

$$
\ddot{\delta}+2 H \dot{\delta}-4 \pi G \rho_{M}=0
$$

so that the dark energy only enters through its effect on the Hubble parameter $H(z)$. As we have checked, $\Delta H / H=1-H / H_{\Lambda C D M}$ decreases from $\simeq 10 \%$ to $\simeq 0$ for $z$ ranging between the beginning $(z \simeq 150)$ and the end $(z \simeq 0.45)$ of the matter dominated era, taking $\left(\Omega_{M}, \Omega_{\Lambda}, h\right)=(0.3,0.7,0.7)$ for the concordance $\Lambda \mathrm{CDM}$ model. As such, we do expect that the growth index $f=d \ln \delta / d \ln a$ is almost the same as the $\Lambda \mathrm{CDM}$ one so guaranteeing a good agreement with this kind of data. However, Eq.(42) is recovered assuming that dark energy does not cluster. While this is reasonable for a negative pressure fluid, this could not be the case for our model since its EoS increases from the negative value $w_{\text {eff }}(z=0.45)=-0.84$ to $w_{\text {eff }}(z=150) \simeq 0.99$ during the matter era. As such, the dark energy sound speed $c_{s}^{2}=\partial p_{D E} / \partial \rho_{D E}$ crosses the dust value $c_{s}=0$. As such, it is likely that dark energy can partecipate to the collapse leading to structure formation thus invalidating Eq.(42) and claiming for a coupled set of equations.

As a final issue, we would like to comment on a nice feature of the model. As it is apparent from Fig. 4 , the presence of a dark energy dominated era before the matter epoch makes it possible to partially solve the coincidence problem. Indeed, the ratio $r(z)=\rho_{M}(z) / \rho_{D E}(z)$ crosses the unit value two times in the universe history. As a consequence, $r(z) / r_{0}$ changes by two order of magnitudes (from 0.1 to 10) over the redshift range $(-0.5,2.5)$. Converting the redshift into the dimensionless time $\tau=t / t_{0}$, we see that $r(z)$ stays close to its present day value over most of the universe history thus significantly alleviating the coincidence problem. 


\section{Fourth order equivalent theory}

The likelihood analysis performed above has successfully demonstrated that the model we have introduced in Sect. 2 is able to reproduce the available astrophysical data. This encouraging result makes us confident that our approach to halting the eternal acceleration through the introduction of a negative cosmological constant is observationally well founded. Nevertheless, a model comprising two dark ingredients (the scalar field $\phi$ and the $\Lambda<0$ term) may be considered as unsatisfactory because of the need to theoretically motivate two rather than one new fluids. Moreover, one of them is an unusual negative cosmological constant which is difficult to reconcile with the classical interpretation in terms of vacuum energy. As such, it is interesting to work out a possible reinterpretation of the model in terms of a radically different approach.

Fourth order theories of gravity have recently attracted a lot of attention as a valid alternative to explain cosmic acceleration without the need of any dark energy fluid. Moreover, it has been developed a quick method to find out a $f(R)$ theory giving the same cosmic dynamics of a given dark energy model. Since all the tests we have considered above rely on the Hubble parameter, it is obvious that the reconstructed $f(R)$ theory will fit the data in the same way as the model discussed up to now.

Here, we first summarize the basics of $f(R)$ theories and the method of reconstructing the gravity Lagrangian from the Hubble parameter $H(z)$ and then present the application to the model we are considering.

\subsection{Basics of $f(R)$ theories}

Much interest has been recently devoted to the so called curvature quintessence according to which the universe is filled by pressureless dust matter only and the acceleration is the result of the modified Friedmann equations obtained by replacing the Ricci scalar curvature $R$ with a generic function $f(R)$ in the gravity Lagrangian. The Friedmann equations therefore read [19, 20] :

$$
\begin{aligned}
& H^{2}=\frac{1}{3}\left[\frac{\rho_{m}}{f^{\prime}(R)}+\rho_{\text {curv }}\right], \\
& 2 \frac{\ddot{a}}{a}+H^{2}=-w_{\text {curv }} \rho_{\text {curv }},
\end{aligned}
$$

where the prime denotes derivative with respect to $R, \rho_{\text {curv }}$ is the energy density of an effective curvature fluid given as:

$$
\rho_{\text {curv }}=\frac{1}{f^{\prime}(R)}\left\{\frac{1}{2}\left[f(R)-R f^{\prime}(R)\right]-3 H \dot{R} f^{\prime \prime}(R)\right\},
$$

and the barotropic factor of the curvature fluid is :

$$
w_{\text {curv }}=-1+\frac{\ddot{R} f^{\prime \prime}(R)+\dot{R}\left[\dot{R} f^{\prime \prime \prime}(R)-H f^{\prime \prime}(R)\right]}{\left[f(R)-R f^{\prime}(R)\right] / 2-3 H \dot{R} f^{\prime \prime}(R)}
$$


Assuming that there is no interaction between matter and curvature terms, the continuity equation for $\rho_{\text {curv }}$ reads [23]:

$$
\dot{\rho}_{\text {curv }}+3 H\left(1+w_{\text {curv }}\right) \rho_{\text {curv }}=\frac{3 H_{0}^{2} \Omega_{M} \dot{R} f^{\prime \prime}(R)}{\left[f^{\prime}(R)\right]^{2}} a^{-3}
$$

which is identically satisfied as can be easily shown using Eq.(43) and expressing the scalar curvature $R$ as function of the Hubble parameter:

$$
R=-6\left(\dot{H}+2 H^{2}\right) \text {. }
$$

Combining Eqs.(43) with Eq.(44) and using the definition of $H$, one finally gets the following master equation for the Hubble parameter [23]:

$$
\dot{H}-\frac{1}{2 f^{\prime}(R)}\left\{3 H_{0}^{2} \Omega_{M} a^{-3}+\ddot{R} f^{\prime \prime}(R)+\dot{R}\left[\dot{R} f^{\prime \prime \prime}(R)-H f^{\prime \prime}(R)\right]\right\} .
$$

Inserting Eq.(48) into Eq.(49), one ends with a fourth order nonlinear differential equation for the scale factor $a(t)$ that cannot be analitically solved also for the simplest cases (for instance, $f(R) \propto R^{n}$ unless dust matter contribution is discarded). Moreover, although technically feasible, a numerical solution of Eq.(49) is plagued by the large uncertainties on the boundary conditions (i.e., the present day values of the scale factor and its derivatives up to the third order) that have to be set to find out $a(t)$ by solving Eq.(49).

Given these mathematical difficulties, a different approach has been proposed in [23] (hereafter CCT) where Eq.(49) is considered as a way to determine $f(R)$ rather than $a(t)$. Rearranging the different terms suitably, CCT obtained a linear third order differential equation for $f$ in terms of the redshift $z=1 / a-1$ (having set $a_{0}=1$ ) that can be easily solved numerically for a given $H(z)$. By this method, it is then possible to find out which $f(R)$ theory reproduces the same dynamics of a given dark energy model, thus showing a formal equivalence between these two radically different approaches.

CCT developed the method using the redshift $z$ as integration variable since it is common to have an analytical expression for the Hubble parameter as function of $z$. However, this is not the case for the model considered here, so that it turns out to be numerically best suited to use $t$ as integration variable. To this aim, we follow [55] to

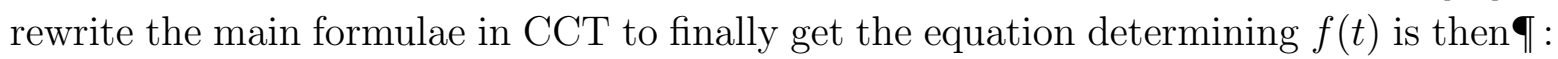

$$
\mathcal{H}_{3}(t) \frac{d^{3} f}{d t^{3}}+\mathcal{H}_{2}(t) \frac{d^{2} f}{d t^{2}}+\mathcal{H}_{1}(t) \frac{d f}{d t}=-3 H_{0}^{2} \Omega_{M} \dot{R}^{2} a^{-3}(t)
$$

with :

$$
\begin{aligned}
& \mathcal{H}_{1}=2 \dot{H} \dot{R}+H \ddot{R}+2 \ddot{R}^{2} \dot{R}^{-1}-d^{3} R / d t^{3} \\
& \mathcal{H}_{2}=-(2 \ddot{R}+H \dot{R}) \\
& \mathcal{H}_{3}=\dot{R}
\end{aligned}
$$

I With an abuse of notation, we write $f(t)$ rather than $f[R(t)]$. 
where $R$ is given by Eq.(48). In order to integrate Eq.(50), we need to specify boundary conditions that are more conveniently assigned at the present time. We slightly generalize here the discussion presented in CCT. First, let us remember that, in a fourth order theory, we may define an effective gravitational constant as $G_{\text {eff }}=G_{N} / f^{\prime}(R)$, with $G_{N}$ the usual Newtonian gravitational constant. Its rate of variation will be given as:

$$
\frac{\dot{G}_{e f f}}{G_{e f f}}=-\frac{1}{t_{0}} \frac{f^{\prime \prime}(R)}{f^{\prime}(R)} \frac{d R}{d \tau} .
$$

It is quite natural to assume that the effective and the Newtonian gravitational couplings take the same values today, so that we get the condition:

$$
f^{\prime}\left(R_{0}\right)=1 .
$$

Evaluating Eq.(54) for $t=t_{0}$ (i.e., $\tau=1$ ), we may determine $f^{\prime \prime}\left(R_{0}\right)$ provided an estimate of $\left(\dot{G}_{e f f} / G_{e f f}\right)_{t=t_{0}}$ is given. Since in our theory $G_{N}$ is constant, we may assume that the measurements of the variation of $G_{N}$ [56] actually refers to $G_{\text {eff }}$ and use these results to get an estimate of $\left(\dot{G}_{e f f} / G_{e f f}\right)_{t=t_{0}}$. We thus take as our boundary condition:

$$
f^{\prime \prime}\left(R_{0}\right)=-t_{0}\left(\frac{\dot{G}_{e f f}}{G_{e f f}}\right)_{o b s}\left(\frac{d R}{d \tau}\right)^{-1},
$$

having used Eq.(55). Finally, inserting Eqs.(55) and (156) into Eq.(45) and then in (43) evaluated today, we get:

$$
\begin{aligned}
f\left(R_{0}\right) & =6 H_{0}^{2}\left(1-\Omega_{M}+\frac{R_{0}}{6 H_{0}^{2}}\right) f^{\prime}\left(R_{0}\right) \\
& +6 H_{0}\left(\frac{d R}{d t}\right)_{t=t_{0}} f^{\prime \prime}\left(R_{0}\right) .
\end{aligned}
$$

From Eqs.(56) - (57), the following boudary conditions straightforwardly descend :

$$
\begin{aligned}
& \left(\frac{d f}{d t}\right)_{t=t_{0}}=\left(\frac{d R}{d t}\right)_{t=t_{0}} f^{\prime}\left(R_{0}\right), \\
& \left(\frac{d^{2} f}{d t^{2}}\right)_{t=t_{0}}=\left(\frac{d R}{d t}\right)_{t=t_{0}}^{2} f^{\prime \prime}\left(R_{0}\right)+\left(\frac{d^{2} R}{d t^{2}}\right)_{t=t_{0}} f^{\prime}\left(R_{0}\right)
\end{aligned}
$$

that have to be used, together with Eq.(57), to numerically solve Eq.(150). Combining the solution thus obtained for $f(t)$ with $R(t)$ evaluated through Eq.(48), one finally finds $f(R)$ thus recovering the higher order theory that mimicks the assigned dark energy model. We refer the reader to [23, 55, 54] for some interesting examples.

A preliminary comment is in order here, however. The dark energy model and its $f(R)$ duality representation share the same expression for the Hubble parameter $H(z)$ (and hence for the scale factor dependence on cosmic time). As such, all the observational tests relying directly on $H(z)$ (as the acoustic peak parameter $\mathcal{A}$ ) or its integral (as the luminosity distance or the age of the universe) are not able 
to discriminate between a whatever dark energy model and its $f(R)$ counterpart. Nevertheless, such an equivalence only holds at the background level. Indeed, since the underlying theory of gravity is radically different, at the perturbative level, the two models make clearly distinct predictions. As a consequence, observational tests relying on the solution of perturbation equations (such as the growth factor and the power spectrum) could be able to break this degeneracy. Actually, at the moment, observational determinations of the growth index $d \ln \delta / d \ln a$ are still quite noisy, while the uncertainties in the relation betwenn the observationally derived galaxy power spectrum and the theoretically predicted matter one still prevent from discriminating among dark energy and modified gravity. Moreover, we only rely here on tests depending on $H(z)$ so that the $f(R)$ theory we will reconstruct is observationally equivalent to our two fluids scenario.

\subsection{Reconstruction of $f(R)$}

All we need to apply the procedure described above is an expression for the Hubble parameter $H$ as function of the cosmic time $t$. For the model we are considering, this may be obtained numerically as described in Sect. 2 so that we only have to choose a value for $\dot{G}_{e f f} / G_{e f f}$ in order to set the initial condition for $f^{\prime \prime}\left(R_{0}\right)$. We fix $\dot{G}_{e f f} / G_{e f f}=0$ in good agreement with most of the estimates in [56]. We have also checked that changing this value within the quoted uncertainties does not affect significantly the main results.

Before presenting the reconstructed gravity Lagrangian, there is a conceptual point to clarify. The method described above represents a sort of bridge between two different scenarios. In particular, their matter contents could be different, so that we should define both $\Omega_{M}^{c u r v}$ and $\Omega_{M}^{D E}$ to denote this quantity in the two different models. In principle, there is no reason why $\Omega_{M}^{c u r v}=\Omega_{M}^{D E}$ should hold. However, since $\Omega_{M}^{D E}$ is close to the fiducial value $\left(\Omega_{M} \simeq 0.3\right)$ suggested by model independent estimates (e.g., from galaxy clusters abundance), we take $\Omega_{M}^{c u r v}=\Omega_{M}^{D E}$. Should we have chosen a different value for $\Omega_{M}^{c u r v}$, we have had a different reconstructed $f(R)$, but still providing the same Hubble parameter as the model with the scalar field and the negative $\Lambda$ we have tested against data. To be more precise, one could say that our method is not able to recover a single $f(R)$, but rather a class of $f(R)$ models parameterized by $\Omega_{M}^{\text {curv }}$. An astrophysics based estimate of $\Omega_{M}$ is the only way to break this degeneracy making it possible to select the most suitable member in this family of fourth order theories.

With this remark in mind, we have performed the reconstruction of $f(R)$ for the model presented in Sect. 2 setting its parameters $\left(\Omega_{M}, \Omega_{\Lambda}, w_{0}\right)$ to their best fit values. Rather than reconstructing $f(R)$ directly, it is more instructive to first consider:

$$
\eta=\left[\frac{f(R)}{R}\right] \times\left[\frac{f(R)}{R}\right]_{z=0}^{-1}
$$

which is identically 1 for the Einstein- Hilbert Lagrangian, $f(R)=R$. Departures of $\eta$ from unity therefore quantifies how much the modified Lagrangian should depart from the standard one in order to fit the data as well as the input Hubble parameter. 


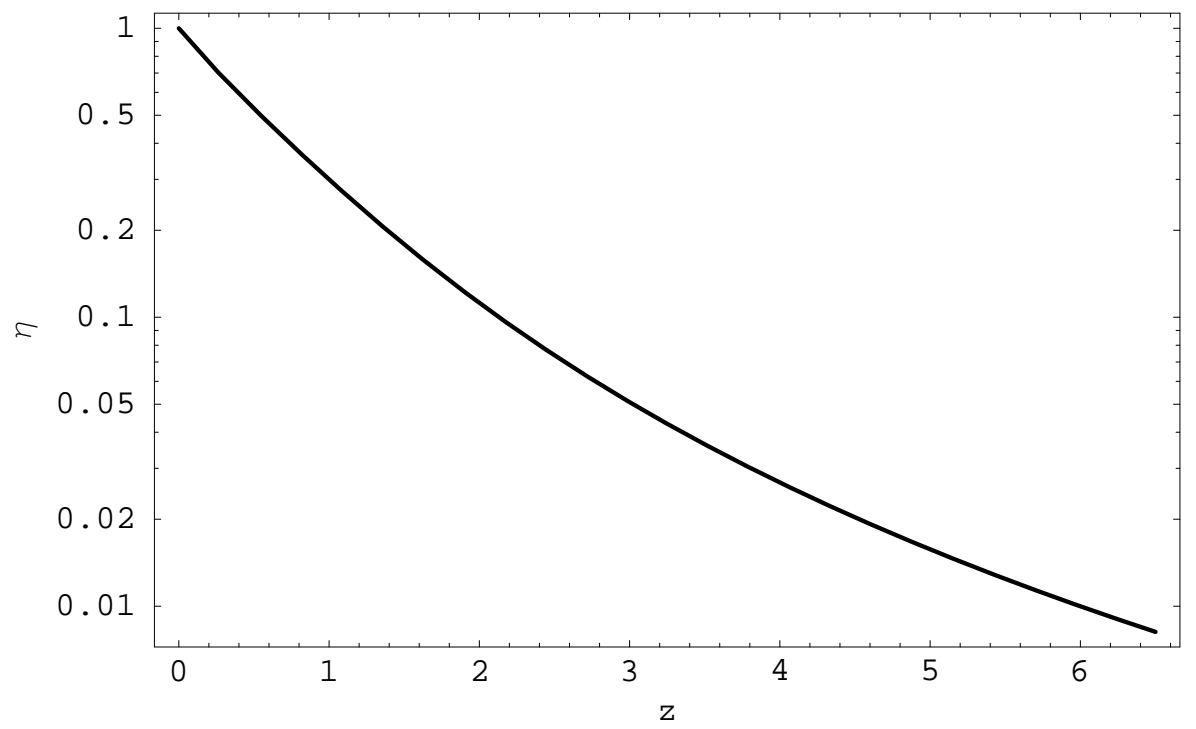

Figure 5. $\eta$ as function of the redshift $z$ for the $f(R)$ reconstructed from the best fit model. Here we define $\eta=[f(R) / R] /[f(R) / R]_{z=0}$, see the text for further details.

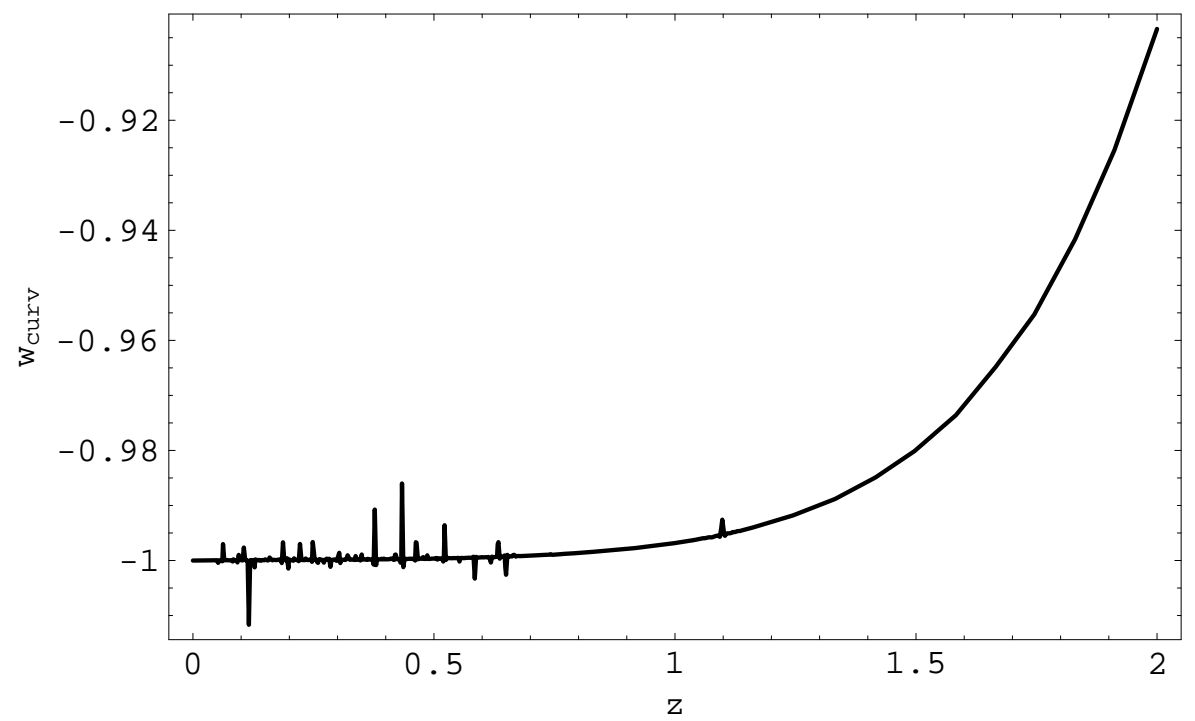

Figure 6. The EoS $w_{\text {curv }}$ of the effective curvature fluid for the reconstructed $f(R)$ theory. Note that the small fluctuations are only artefacts due to numerical noise.

To this aim, it is interesting to look at Fig. 5 where we report $\eta$ as function of $z$ over the redshift range $0 \leq z \leq 6$, extending well above the one probed by the data we have used. It is apparent that $\eta$ quickly departs from 1, i.e. the reconstructed $f(R)$ dramatically differs from the Einsteinian one. This is not unexpected at all. Indeed, in order to fit the data with a matter only universe, one should strongly modifies the gravity Lagrangian at least for values of $R_{s}=R / R_{0}$ corresponding to the accelerating expansion era. For larger values of $z$, the universe is decelerating so that one could naively expect to recover a standard situation with $\eta$ approaching 1 . This is clearly not the case. 
Actually, this somewhat counterintuitive result is easily explained reconsidering Fig.4 which shows that in our model dark energy dominates again after a finite period of matter domination. In order to recover this unusual behaviour, $f(R)$ must still depart from the General Relativity Lagrangian.

Although the reconstructed $f(R)$ is obtained numerically, it is useful to have an analytical approximation which we indeed find to be given by:

$$
f(R)=f_{0} R_{s}\left[1+\left(\alpha R_{s}^{n}+\beta R_{s}^{-m}\right) \ln R_{s}\right]
$$

with $R_{s}=R / R_{0},(\alpha, \beta, n, m)$ real parameters to be fitted to the numerical data. We have checked that such a formula reproduces the reconstructed $f(R)$ within less than $4 \%$ over the full redshift range we have probed. For the best fit model, we get:

$$
(\alpha, \beta, n, m)=(-0.38,-0.61,-0.15,0.70) .
$$

Changing the values of $\left(\Omega_{M}, \Omega_{\Lambda}, w_{0}\right)$ alters these values, but Eq.(61) still provides a very good approximation. We stress, however, that the approximating formula (61) has been tested only over the redshift range $(0,6)$ so that cannot be extrapolated to larger values. As such, one has not to give any weight to the fact that $f(R)$ does not reduce to $R$ in the very early universe as expected to recover the BBN succesfull results.

It is interesting to consider the EoS $w_{\text {curv }}$ of the effective curvature fluid which is plotted in Fig.6. This plot shows that the curvature fluid actually behaves as a sort of usual cosmological constant with its EoS increasing with resdshift, but still remaining quite close to the $\Lambda$ value $w=-1$ over the full redshift range probed by the data. This result could be somewhat anticipated noting that the reconstructed $f(R)$ theory provide the same $H(z)$ and hence the same $\Delta H / H$ as the starting model. Since this quantity stays within $10 \%$ over the range $0 \leq z \leq 6$, it is not surprising that the effective curvature fluid behaves as an almost $\Lambda$ term. However, we have checked that $w_{\text {curv }}$ departs more and more from $w=-1$ as $z$ increases even if we are not able to draw definitive conclusions because of the dramatical increase of the numerical noise.

As a final comment, it is worth stressing again that the reconstructed $f(R)$ provides the same cosmic dynamics (i.e., scale factor and Hubble parameter) of a model comprising both a quintessential scalar field and a negative cosmological constant. As a consequence, being the background dynamics exactly the same, the $f(R)$ model based on Eq.(61) predicts the same behaviour for the luminosity distance as function of the redshift $z$ (and hence the same SNeIa Hubble diagram and gas mass fraction vs $z$ curve) and equal numerical values for the acoustic peak and shift parameters. As such, all the observational tests in Sect. 3 successfully met by the negative $\Lambda$ model are equally satisfied by its $f(R)$ duality representation constructed here. Such a fourth order theory is therefore able to both fit the available data and avoid eternal acceleration. In particular, the net effect is to mimic an unusual negative cosmological constant with a modified gravity Lagrangian and no dark energy. This interesting result shows that a $\Lambda<0$ term could result as a net effect of forcing the gravity theory to be an Einsteinian rather than a fourth order one. Moreover, halting eternal acceleration is therefore possible without adding any ad hoc ingredient to the cosmic pie. 


\section{Conclusions}

The unprecedented high quality data accumulated in the recent years have depicted the scenario of a spatially flat universe presently undergoing a phase of accelerated expansion thus motivating the lot of interest devoted to the search of viable candidates to drive this cosmic acceleration. Although many possible models have been put on the ground, almost all of them predict a two phase scenario with standard dust matter dominating the first epoch of decelerated expansion and a (actual or effective) dark energy fluid fueling the present day cosmic speed up. Notwithstanding the underlying mechanism, the fate of the universe seems to be yet written. As matter fades away, dark energy becomes more and more dominant leading to an eternal acceleration $\dagger$ so that serious problems arise with the formulation of the $\mathcal{S}$ matrix and quantum field theory.

In an attempt to avoid these problems, we have explored here a model made out of three rather than two ingredients. Besides the usual dust matter and radiation and a scalar field responsible of cosmic acceleration, a negative cosmological constant has been added to the energy density budget. While being subdominant during the matter dominated decelerated phase and the scalar field dominated accelerated epoch, this new component may contrast the action of the quintessential scalar field during the future evolution of the universe. Depending on the balance between these two terms, eternal acceleration may be avoided leading to a Big Crunch collapse in a finite time. This possibility renders the model quite attractive from a theoretical point of view and have motivated us to further explore its viability through a comparison with the available astrophysical data. To this aim, we have fitted the model to the most recent release of SNeIa data (made out of data coming from the SNLS, ESSENCE and GOODS surveys) and the gas mass fraction in galaxy clusters also setting a prior on the acoustic peak and shift parameters. This large dataset has been successfully reproduced thus showing that the presence of a negative $\Lambda$ is fully consistent with the data. As such, our model may be considered as a viable solution to the problem of eternal acceleration.

It is, however, worth investigating whether the same model may be interpreted as an effective representation of a radically different scenario. Such a possibility is offered by fourth order theories of gravity according to which cosmic acceleration is the result of a modified gravity Lagrangian with the scalar curvature $R$ replaced by an analytic function $f(R)$ to be determined by the data. Using a method developed by one of us [23, 55], we have reconstructed $f(R)$ by imposing that the cosmic dynamics is the same as the one determined by the action of the scalar field and the negative $\Lambda$. Since the Hubble parameter is the same in both scenarios, both models are able to fit the data in the same way. Moreover, the reconstructed $f(R)$ does not include any cosmological constant term thus showing that a negative $\Lambda$ could also be the consequence of forcing a fourth order Lagrangian to the linear Einsteinian one.

\footnotetext{
+ Here, we are not considering phantom dark energy which leads to a superaccelerated expansion ending in a Big Rip like singularity. Note, however, that phantom scenarios are affected by the same problems of eternal acceleration till the cosmic doomsday.
} 
Although encouraging, these results must be considered only preliminary. Indeed, the model is affected by some undesirable shortcomings when applied to the early universe. First, the model turns out to be matter dominated only for a finite redshift range, while its expansion is scalar field driven during most of the high redshift range. Moreover, the scalar field EoS converges towards the stiff matter value $w=1$ so that one could worry about the growth of structure and the CMBR anisotropy spectrum. Both these results are not fully unexpected. Indeed, it has yet been demonstrated that an exponential potential may lead towards a scalar field dominated universe. Our result shows that this conclusion also holds in the case a negative $\Lambda$ is present as could be predicted in advance noting that this term becomes quickly subdominant at large $z$ with respect to the matter and the scalar field ones. One could therefore consider the possibility to replace the exponential potential with a different $V(\phi)$ using, e.g., the sum of two exponential with opposite signs. Suitably weighting the two terms may lead to a model which is similar to the present one at small $z$ thus giving the same successful match with observations. On the other hand, making the additional term dominant for large $z$ may solve the problems of the present model in the early universe. We stress, however, that here we have been more interested in looking for a model able to halt eternal acceleration. We have therefore paid more attention to the recent and future universe so that some problems with the early universe were foreseeable. The complicated task of matching the early, late and future expansion looking for a suitable potential will be addressed in a forthcoming work.

Further developments of the approach pursued here are possible from the observational and the theoretical point of view. On one hand, we have only tested the dynamics of the model, but severe constraints may be obtained investigating structure formation (see, e.g., 57] for a discussion of this issue for the expoential potential model). To this aim, a first important step may be done solving the perturbation equation in the linear regime thus determining the growth factor $D=\delta / a$ and the growth index $f=d \ln \delta / d \ln a$. Since a negative $\Lambda$ has the same effect of an attractive gravitational potential, it is possible that the collapse of structures is enhanced so that it is worth investigating what constraints may be imposed by requiring that this effect does not change significantly neither $D$ nor $f$ with respect to the successful $\Lambda$ CDM predictions. It is worth stressing, at this point, that which are the perturbation equations to solve depend on the scenario adopted. Should we interpret the model in terms of $f(R)$ theories, the standard theory of linear perturbations must be abandoned and different equations have to used. This gives us the possibility to select between the two equivalent descriptions retaining the one which predicts $D(a)$ and $f(a)$ most similar to the $\Lambda$ CDM ones since they are more likely able to fit the large scale structure data.

On a different ground, one can arguably discriminate between the two components dark energy model and its $f(R)$ duality representation based on Solar System tests. Indeed, in the low energy limit, the static and spherically symmetric solution of the Einstein equations for the two components dark energy model leads to the usual Newtonian potential (apart some negligible deviations introduced by the tiny neative $\Lambda$ 
and the scalar field) so that all the classical tests of gravity are automatically verified. On the other hand, the low energy limit of $f(R)$ theories may lead to significant deviations from the Newtonian potential. As such, these theories are indeed severely constrained by the Solar System tests so that some models have been carefully designed just to evade this problem [58]. Although a strong debate is still present on which is the the correct way to work out the low energy limit of such theories, it is worth investigating this issue with some detail in a future work in order to see whether our approximated $f(R)$ expression (61) is indeed viable from this point of view. Should this not be the case, we could give it away, but still retaining the two components dark energy model as a viable way to halt eternal acceleration.

A more philosophical comment is in order. Along the work presented here, we have first introduced a two components dark energy model comprising a negative $\Lambda$ and an exponential potential scalar field $\phi$. After successfully testing it against the data, we have worked out its fourth order counterpart determining the function $f(R)$ entering the modified gravity Lagrangian giving the same cosmic expansion $H(z)$. A guidance idea has been the Occam's razor that suggests to give out models with an unnecessary large number of unknown elements. The price to pay has been to replace the simple general relativistic $f(R)=R$ with a fourth order expression analytically approximated as in Eq.(61). One could wonder whether such a complicated Lagrangian may indeed be in line with the Occam's razor spirit. Actually, we are at the moment unable to find out a fundamental theory leading to our reconstructed $f(R)$, although some Lagrangian containing the sum of terms with different powers of $R$ have been studied in literature (see, e.g., third reference in [22]). Should a fundamental theory not be found, one can indeed consider unnecessary our modification of $f(R)$ and put our theory on the same ground as the two components dark energy model we have started from.

As a conclusive remark, we believe it is worth stressing that halting eternal acceleration is possible. Using a two component model comprising a quintessential scalar field and a negative $\Lambda$ represents the easiest way to generate a transient cosmic acceleration. Fourth order theories make it possible to interpret both these (somewhat problematic) terms as effective manifestations of a different scenario. The reconstructed $f(R)$ may then be seen as the missing bridge between the easy world of many components models and the mathematically complicated structure of higher order gravity theories.

\section{Acknowledgments}

VFC is grateful to S. Capozziello and A. Troisi for the interesting discussions on the manuscript and to V. Salzano for help with the MCMC programming.

\section{References}

[1] S. Perlmutter et al., ApJ, 483, 565, 1997; A.G. Riess et al., AJ, 116, 1009, 1998; B.P. Schmidt et al., ApJ, 507, 46, 1998; P.M. Garnavich et al., ApJ, 509, 74, 1998; S. Perlmutter et al., ApJ, 
$517,565,1999$

[2] A.G. Riess et al., ApJ, 607, 665, 2004

[3] C.L. Bennett et al., ApJS, 148, 1, 2003; D.N. Spergel et al., ApJS, 148, 175, 2003

[4] R. Rebolo et al., MNRAS, 353, 747, 2004

[5] S. Dodelson et al., ApJ, 572, 140, 2002; W.J. Percival et al., MNRAS, 337, 1068, 2002; A.S. Szalay et al., ApJ, 591, 1, 2003; E. Hawkins et al., MNRAS, 346, 78, 2003; A.C. Pope et al., ApJ, 607, 655,2004

[6] P.J.E. Peebles, B. Rathra, Rev. Mod. Phys., 75, 559, 2003; T. Padmanabhan, Phys. Rept., 380, 235, 2003

[7] S.M. Carroll, W.H. Press, E.L. Turner, ARAA, 30, 499, 1992; V. Sahni, A. Starobinski, Int. J. Mod. Phys. D, 9, 373, 2000

[8] M. Tegmark et al., Phys. Rev. D, 69, 103501, 2004

[9] U. Seljak et al., Phys, Rev. D, 71, 043511, 2005

[10] M. Tegmark et al., Phys. Rev. D, 74, 123507, 2006

[11] M. Sasaki, H. Suzuki, K. Yamamoto, J. Yokoyama, Class. Quantum Grav. 10, L55, 1993

[12] M.B. Green, J.H. Schwarz, E. Witten, Superstring theory, vols I and II, Cambridge University Press, Cambridge, 1987

[13] T. Banks, W. Fischler, hep-th/0102077, 2001; S. Hellerman, N. Kaloper, L. Susskind, JHEP, 0106, 003, 2001; W. Fischler, A. Kashani-Poor, R. McNees, S. Paban, JHEP, 0107, 003, 2001; P.O. Mazur, E. Mottola, Phys. Rev. D, 64, 104022, 2001; J. Ellis, N.E. Mavromatos, D.V. Nanopoulos, hep-th/0105206, 2001; E. Witten, hep-th/0106109, 2001

[14] F. David, Mod. Phys. Lett. A, 3, 1651, 1988; J. Distler, H. Kawai, Nucl. Phys. B, 321, 509, 1989; N.E. Mavromatos, J.L. Miramontes, Mod. Phys. Lett. A, 4, 1847, 1989; E. D’Hoker, P.S. Kurzepa, Mod. Phys. Lett. A, 5, 1411, 1990

[15] J.A. Gu, W.Y.P. Hwang, Phys. Rev. D, 73, 023519, 2006

[16] R. Cardenas, T. Gonzalez, Y. Leiva, O. Martin, I. Quiros, Phys. Rev. D, 67, 083501, 2003

[17] J. Grande, J. Sola, H. Stefancic, JCAP, 0608, 011s, 2006

[18] S. Weinberg, Rev. Mod. Phys., 61, 1, 1989, and references therein

[19] S. Capozziello, Int. J. Mod. Phys. D, 11, 483, 2002

[20] S. Capozziello, S. Carloni, A. Troisi, Recent Research Developments in Astronomy and Astrophysics, Research Signpost Publisher, astro-ph/0303041, 2003

[21] S. Capozziello, V.F. Cardone, S. Carloni, A. Troisi, Int. J. Mod. Phys. D, 12, 1969, 2003; S. Carloni, P.K.S. Dunsby, S. Capozziello, A. Troisi, Class. Quant. Grav., 22, 4839, 2005; S. Capozziello, V.F. Cardone, M. Francaviglia, Gen. Rel. Grav., 38, 711, 2006

[22] S. Nojiri, S.D. Odintsov, Phys. Lett. B, 576, 5, 2003; S. Nojiri, S.D. Odintsov, Mod. Phys. Lett. A, 19, 627, 2003; S. Nojiri, S.D. Odintsov, Phys. Rev. D, 68, 12352, 2003; S.M. Carroll, V. Duvvuri, M. Trodden, M. Turner, Phys. Rev. D, 70, 043528, 2004

[23] S. Capozziello, V.F. Cardone, A. Trosi, Phys. Rev. D, 71, 043503, 2005

[24] C. Rubano, P. Scudellaro, Gen. Rel. Grav., 34, 307, 2001

[25] R. Cardenas, T. Gonzalez, O. Martin, I. Quiros, D. Villegas, Gen. Rel. Grav., 34, 1877, 2002

[26] M. Pavlov, C. Rubano, M. Sazhin, P. Scudellaro, ApJ, 566, 619, 2002; C. Rubano, M. Sereno, MNRAS, 335, 30, 2002; M. Demianski, E. Piedipalumbo, C. Rubano, C. Tortora, A\&A, 431, 27,2005

[27] R. de Ritis et al., Phys. Rev. D, 42, 1091, 1990; S. Capozziello, R. de Ritis, C. Rubano, P. Scudellaro, Riv. Nuovo Cimento, 19, 1, 1996; R. de Ritis, A.A. Marino, C. Rubano, P. Scudellaro, Phys. Rev. D, 62, 043506, 2000

[28] M. Fukugita and P.J.E Peebles, ApJ, 616, 643, 2004

[29] T. Davis et al., ApJ, 666, 716, 2007

[30] P. Astier et al., A\&A, 447, 31, 2006

[31] W.M. Wood - Vassey et al., ApJ, 666, 694, 2007

[32] A.G. Riess et al., ApJ, 659, 98, 2007 
[33] S. Sasaki, PASJ, 48, L119, 1996; U. Pen, New Ast., 2, 309, 1997

[34] S.W. Allen, R.W. Schmidt, A.C. Fabian, MNRAS, 334, L11, 2002; S.W. Allen, R.W. Schmidt, S. Bridle, MNRAS, 346, 593, 2003

[35] S.W. Allen, R.W. Schmidt, H. Ebeling, A.C. Fabian, L. van Speybrock, MNRAS, 353, 457, 2004

[36] V. Eke, J.F. Navarro, C.S. Frenk, ApJ, 503, 569, 1998

[37] S.W. Allen, et al. 2007

[38] D. Kirkman, D. Tyler, N. Suzuki, J.M. O’Meara, D. Lubin, ApJS, 149, 1, 2003

[39] D. Eisenstein et al., ApJ, 633, 560, 2005

[40] M.A. Strauss et al., AJ, 124, 1810, 2002

[41] D.N. Spergel et al., ApJS, 170, 377, 2007; see also the tables accessible from the web site http://lambda.gsfc.nasa.gov

[42] J.R. Bond, G. Efstathiou, M. Tegmark, MNRAS, 291, L33, 1997

[43] W. Hu, N. Sugiyama, ApJ, 471, 542, 1996

[44] Y. Wang, P. Mukherjee, ApJ, 650, 1, 2006

[45] W.L. Freedman et al., ApJ, 553, 47, 2001

[46] L.L.R. Williams, P. Saha, AJ, 119, 439, 2000; V.F. Cardone, S. Capozziello, V. Re, E. Piedipalumbo, A\&A, 379, 72, 2001; V.F. Cardone, S. Capozziello, V. Re, E. Piedipalumbo, A\&A, 382, 792, 2002; C. Tortora, E. Piedipalumbo, V.F. Cardone, MNRAS, 354, 353, 2004; T. York, I.W.A. Browne, O. Wucknitz, J.E. Skelton, MNRAS, 357, 124, 2005

[47] J.P. Hughes, M. Birkinshaw, ApJ, 501, 1, 1998; R. Saunders et al., MNRAS, 341, 937, 2003; R.W. Schmidt, S.W. Allen, A.C. Fabian, MNRAS, 352, 1413, 2004

[48] M. Chevallier and D. Polarski, Int. J. Mod. Phys. D, 10, 213, 2001; E.V. Linder, Phys. Rev. Lett., 90, 091301, 2003

[49] E.L. Wright, ApJ, 664, 633, 2007

[50] Ø. Elgarøy, T. Multamäki, A\&A, 471, 65, 2007

[51] L. Krauss, B. Chaboyer, Science, 299, 65, 2003

[52] R. Cayrel et al., Nature, 409, 691, 2001

[53] A. Shafieloo, MNRAS, 380, 1573, 2007

[54] T. Multamaki, I. Vilja, Phys. Rev. D, 73, 024018, 2006

[55] S. Capozziello, V.F. Cardone, E. Piedipalumbo, C. Rubano, Class. Quant. Grav., 23, 1205, 2006

[56] J.P. Uzan, Rev. Mod. Phys., 75, 403, 2003

[57] J.J. Halliwell, Phys. Lett. B, 185, 341, 1987; J. Yokoyama and K. Maeda, Phys. Lett. B, 207, 31, 1988; E.J. Copeland, A.R. Liddle, D. Wands, Phys. Rev. D, 57, 4686, 1998

[58] A.A. Starobinski, JETP Lett., 86, 157, 2007; W. Hu and I. Sawicki, Phys. Rev. D, 76, 064004, 2007; S.A. Applebye and R.A. Battye, Phys. Lett. B, 654, 7, 2007; S. Tsujikawa, Phys. Rev. D, $77,023507,2008$ 